\title{
Spaceborne MIMO Synthetic Aperture Radar for Multimodal Operation
}

\author{
Jung-Hyo Kim, Member, IEEE, Marwan Younis, Senior Member, IEEE, \\ Alberto Moreira, Fellow, IEEE, and Werner Wiesbeck, Fellow, IEEE
}

\begin{abstract}
In this paper, we introduce a novel multiple-input multiple-output (MIMO) synthetic aperture radar (SAR) concept for multimodal operation. The proposed system employs waveforms based on the orthogonal frequency division multiplexing (OFDM) technique and digital beamforming (DBF) on receive. Thereby, it becomes feasible to maximize spatial degrees of freedom, which are necessary for the multimodal operation. The proposed MIMO SAR system produces multiple high-resolution wide-swath SAR imageries that are used for coherent postprocessing. Through this paper, we aim to open up a new perspective of using MIMO concept for a wide-swath SAR imaging with high resolution in interferometric and polarimetric modes, based on OFDM and DBF techniques. Therefore, this paper encompasses broad theoretical backgrounds and general system design issues as well as specific signal processing techniques and aspects.
\end{abstract}

Index Terms-Digital beamforming (DBF), high-resolution wide swath (HRWS), multichannel synthetic aperture radar (SAR) processing, multiple-input multiple-output (MIMO), orthogonal frequency division multiplexing (OFDM) waveform, reconfigurable SAR system, SAR.

\section{INTRODUCTION}

$\mathbf{S}$ YNTHETIC aperture radar (SAR) systems have been extensively contributing to diverse scientific applications and to technological progress in radar technology [1]. Expanding the performance of SAR systems, capabilities of frequent monitoring, wide coverage, and fine geometric resolution become more crucial for future spaceborne SAR missions. However, the performance of conventional single-antenna systems is limited by the fundamental antenna constraint, which allows either a high azimuth resolution or a wide swath width [2]. Consequently, multichannel SAR concepts have emerged as a new paradigm for future spaceborne SAR systems [3]-[5]. The use of multiple receive antennas in SAR systems leads

Manuscript received February 7, 2014; revised May 27, 2014; accepted July $2,2014$.

J.-H. Kim is with Airbus Defence and Space, 88039 Friedrichshafen, Germany (e-mail: jung-hyo.kim@astrium.eads.net).

M. Younis is with the Microwaves and Radar Institute, German Aerospace Center (DLR), Oberpfaffenhofen, 83224 Wessling, Germany (e-mail: marwan. younis@dlr.de).

A. Moreira is with the Microwaves and Radar Institute, German Aerospace Center (DLR), 82230 Oberpfaffenhofen, Germany, and also with the Karlsruhe Institute of Technology, 76131 Karlsruhe, Germany.

W. Wiesbeck is with the Institute of High Frequency Technique and Electronics, Karlsruhe Institute of Technology, 76049 Karlsruhe, Germany.

Color versions of one or more of the figures in this paper are available online at http://ieeexplore.ieee.org.

Digital Object Identifier 10.1109/TGRS.2014.2360148 to a considerable improvement in system performance when compared to the state-of-the-art SAR, and its benefits can be maximized when combined with digital beamforming (DBF) capability [5]. So, SAR systems with DBF functionality on receive are spotlighted as a promising way toward the next generation of SAR instrument [6]-[10]. The high-resolution wide-swath (HRWS) SAR is a representative example of the DBF SAR concept. The HRWS SAR system enables one to circumvent the fundamental limitation in the SAR design and produces high-resolution images over a wide area with the aid of DBF [11], [12]. The multiple channels of the HRWS system provide increased degrees of freedom that are fully exploited for the suppression of azimuth ambiguities caused by lower pulse repetition frequency (PRF) than the Nyquist sampling requirement. The DBF techniques make the ambiguity suppression flexible and adaptive [7], [13]. In this paper, we attempt to build a reconfigurable SAR system concept in which the HRWS SAR imaging operation can be effectively combined with other operation modes, avoiding performance degradation. For this purpose, one needs to increase the spatial degrees of freedom. We achieve it by adding transmit antennas to the multiple receive antennas, the so-called multiple-input multiple-output (MIMO) SAR.

The MIMO concept was proposed by Paulraj and Kailath in the early 1990s for the purpose of increasing channel capacity in wireless communications [14]. This multiple antenna concept has been showing great potential for various applications, not only for wireless communications but also for radar sensors. It has triggered active research work on the multiple antenna systems. Consequently, a radar system with multiple antennas, called MIMO radar, is gaining popularity in the radar community [15]-[21]. The MIMO concept is also very attractive for SAR. In a side-looking scenario, MIMO antennas arranged in along-track produce more spatial samples in azimuth, which can be used for HRWS imaging or ground moving target indication (GMTI). In [22]-[24], an airborne MIMO SAR approach combined with cyclic antenna switching mode and multispectral waveforms was investigated for GMTI. This system allows an improvement of signal-to-clutter-noise ratio based on space-frequency diversity. The MIMO antennas also inspired a new 3-D radar imaging concept, which is called ARTINO [25]. In this concept, a large MIMO array mounted under the wings of an airplane or unmanned aerial vehicle is used to illuminate in the nadir direction and to obtain a fine across-track resolution by DBF. In [26] and [27], techniques to improve spatial resolution have been proposed by employing MIMO antennas along track and across track, respectively. 
Similarly, the MIMO theory could be applied to inverse-SAR imaging for the cross-range resolution enhancement [28]. However, due to complex requirements for the SAR waveform, it was difficult to fully exploit degrees of freedom provided by MIMO configuration for various SAR applications.

In this paper, by employing orthogonal frequency division multiplexing (OFDM) chirp waveforms (cf. [29]) and DBF, we establish the multimodal operation concept of spaceborne MIMO SAR that basically combines advanced HRWS SAR imaging mode with interferometric and polarimetric data acquisition on a single system. In operation, adjusting MIMO antenna constellation, multiple complex SAR image data can be simultaneously obtained for different SAR applications. Furthermore, suppressing ambiguities using the multichannel data contributes to SAR image quality enhancement synchronistically. As aforementioned, special attention should be paid to orthogonal waveforms for the multiple transmission. Due to distributed-target characteristics, the orthogonality of waveforms is the most important issue in realizing the present MIMO SAR concept. On the other hand, the present system should be also capable of producing multiple SAR images for coherent polarimetric and interferometric processing. This means that, although multiple orthogonal waveforms are used to acquire multiple data sets, SAR images reconstructed from individual waveforms must be coherent. These paradoxical conditions cannot be satisfied by conventional coding techniques and frequency diversity schemes using multiple subbands [30]. From this perspective, the MIMO SAR concept presented in this paper is unique.

This paper is organized as follows. Prior to dealing with concrete MIMO SAR techniques, we start with an overview of the OFDM chirp waveform scheme in Section II. In Section III, we present overall MIMO SAR processing steps and the multimodal operation principle in a wider sense, which shows the uniqueness of this approach to overcome the limitations of current systems and achieve the multifunctionality required. In Sections IV and V, we present extended studies about the OFDM waveform performance in the presence of Doppler shift and design an example MIMO SAR system based on the proposed techniques, respectively. The designed system performance is evaluated in terms of ambiguities and sensitivity. We conclude this paper in Section VI with future activities.

\section{OVERVIEW OF OFDM CHIRP WAVEFORM}

The OFDM chirp waveform is essential for the aimed multimodal operation. Assuming knowledge of the OFDM chirp scheme in [29], we give a short overview of the OFDM chirp modulation scheme and point out important properties of the OFDM waveforms.

\section{A. Principle}

The idea behind the new waveform is to use multiple subcarriers for producing nonoverlapping spectra, as shown in Fig. 1. The first row shows the input data sequence, which is a chirp of duration $T_{p}$. The following rows in Fig. 1 show the subcarrier assignment for each of the orthogonal waveforms in

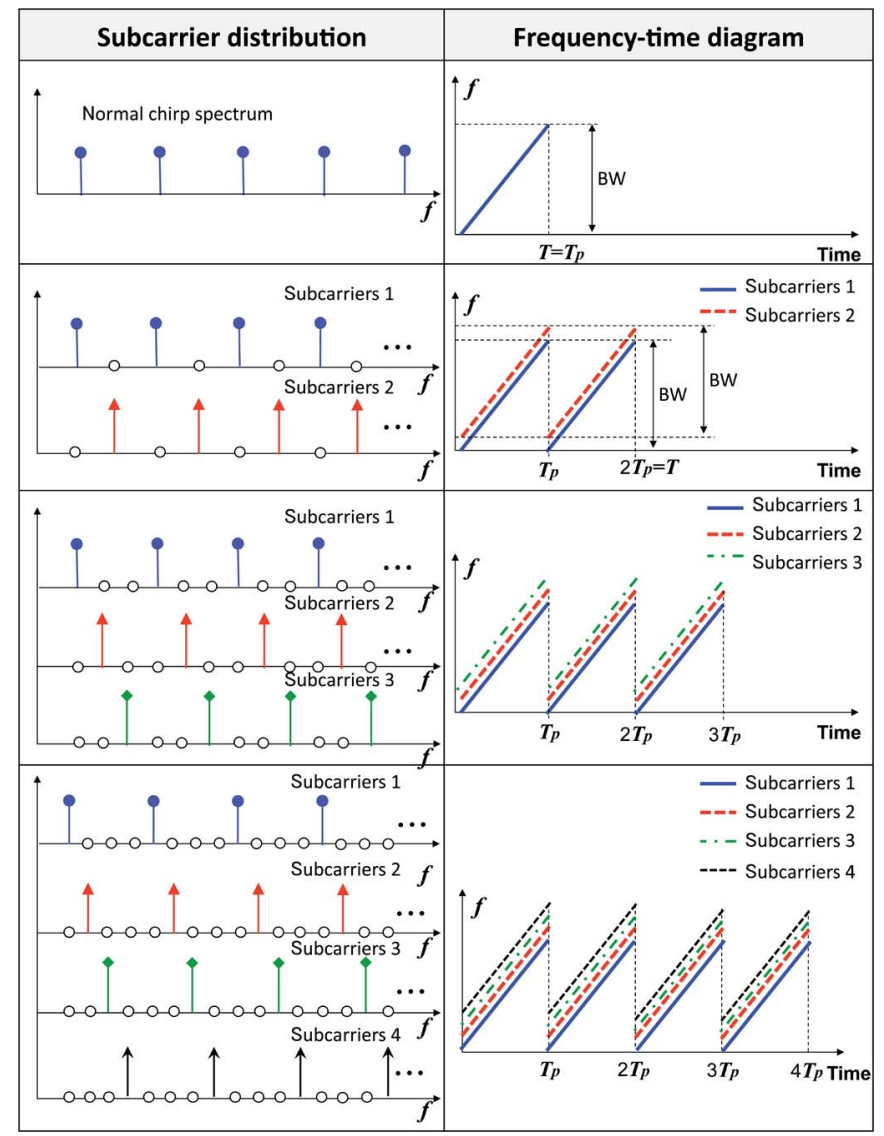

Fig. 1. Subcarrier distribution for orthogonality and corresponding timefrequency diagram of the modulated signals in time domain. By increasing zeros between the subcarriers, one can produce more orthogonal chirplike waveforms at the cost of the chirp repetition. It is noted that a small shift in the time-frequency diagram represents the frequency offset between waveforms.

frequency domain (left) and the corresponding time-frequency diagram (right). Clearly, the subcarriers are orthogonal, since the multiplication of their respective spectra is zero. More orthogonal waveforms are produced by placing additional zeros between the subcarriers, where each new orthogonal waveform causes an increase of the pulse duration by $T_{p}$ [31]. If $M_{\mathrm{Tx}}$ transmitters are used, the OFDM chirp length becomes

$$
T=M_{\mathrm{Tx}} \cdot T_{p}
$$

Due to the zero interleaving, the peak power level of the waveforms will be reduced. However, keeping in mind that the pulse duration is increased, it can be shown that the total energy of the input sequence is conserved according to Parseval's theorem [31]. This repetition property restricts ground coverage regarding range ambiguities. This effect is depicted in Fig. 2. Assume two point targets leading to the delay time difference of $T_{p}$. Due to the repetition property of the OFDM chirp pulse, the impulse response function (IRF) of each point target also has two peaks with $T_{p}$ distance, and IRFs of both echo signals overlap, i.e., ambiguity. Consequently, the maximum delay length of a single subswath must not exceed $T_{p}$ in the aforementioned example. This problem can be solved by DBF on receive as spatial filtering. Detailed principles are introduced in [29]. 

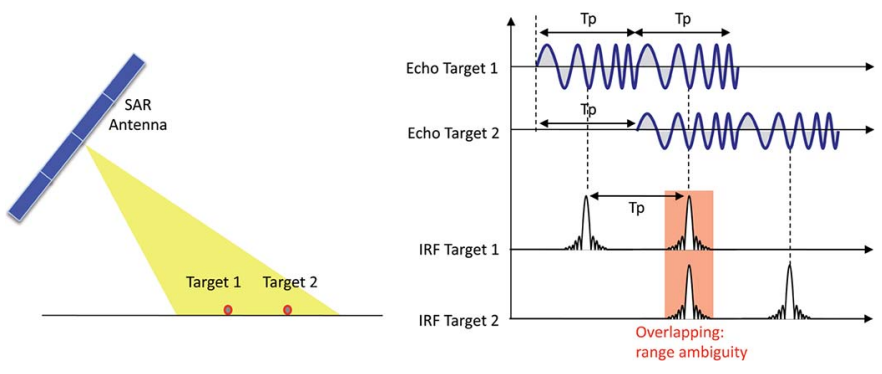

Fig. 2. Allowed maximum delay length for OFDM chirp demodulation. The second peak of target 1 is overlapping with the first peak of target 2 .

The aforementioned scheme could be applied to any waveform. Here, we choose a conventional chirp which allows exploiting its useful characteristics, specifically the constant envelope in both time and frequency domains. This characteristic is the most important property for spaceborne SAR since the flat signal envelope and spectrum are necessary for the maximum power efficiency on transmit and signal-to-noise ratio (SNR), respectively [32], [33].

\section{B. Orthogonality}

The orthogonality of the OFDM chirp waveforms is conserved, if the length of the discrete Fourier transform (DFT) used for modulation on transmit and demodulation on receive is the same of the OFDM signal itself. For an OFDM pulse of duration $T$, the orthogonality can be defined as

$$
\int_{-\infty}^{\infty} s_{i}(t) \cdot s_{j}^{*}\left(\langle t+\tau\rangle_{T}\right) \mathrm{d} t=0 \quad \forall t \in[0, T] \text { and } \tau \in \mathbb{R}
$$

where $i \neq j$ and $\langle\cdot\rangle_{T}$ denotes the arithmetic modulo $T$, which means that $s_{i, j}(t)$ is periodic in $T$. For instance, $T=2 T_{p}$ for a two-transmit-antenna case. Let $T=N \cdot t_{s}$, where $t_{s}$ is the sampling interval. The DFT length is then $N$. Since $s_{i, j}$ is assumed to be cyclic with the period $N$, (2) becomes a cyclic cross-correlation [31]. Therefore, the Fourier transform of the cross-correlation between two OFDM signals is obtained by

$$
\mathcal{F}\left\{s_{i}(t) * s_{j}^{*}(t)\right\}_{N}=\left(S_{i}(f) \cdot S_{j}^{*}(f)\right)_{N}
$$

where $\mathcal{F}\{\}$ denotes the Fourier transform operator. The OFDM signals are modulated by nonoverlapping subcarriers, as shown in Fig. 1. Consequently, the product of two OFDM spectra is zero

$$
\left(S_{i}(f) \cdot S_{j}^{*}(f)\right)_{N}=0 .
$$

Fig. 3 shows the orthogonality of the two OFDM chirp waveforms that was simulated in the presence of speckle, i.e., a typical distributed-target scenario. The OFDM chirp waveforms provide approximately the $-60-\mathrm{dB}$ cross-correlation level for the distributed target, which demonstrates the high potential in using OFDM signals for spaceborne SAR imaging.

Equation (2) tells us that received signals must also be within the OFDM pulse duration $T$, a value that is typically exceeded in spaceborne SAR scenarios due to the large delays associated

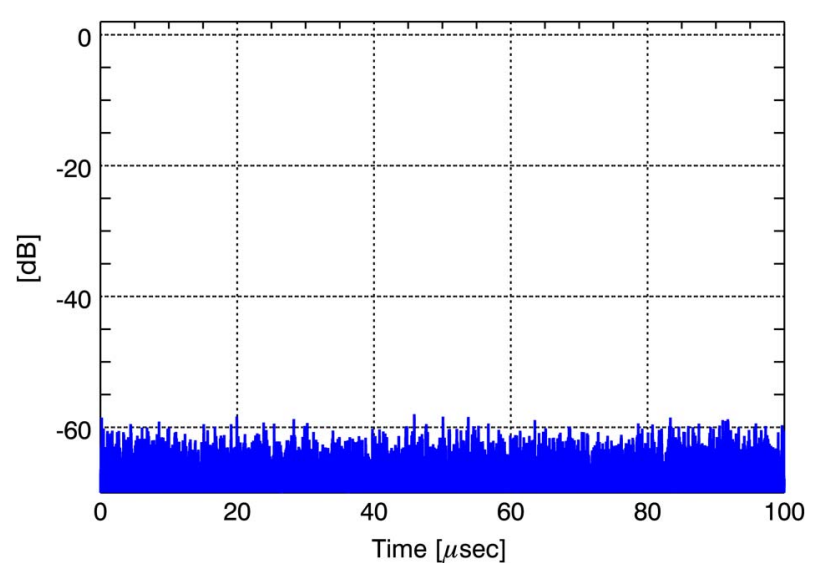

Fig. 3. Cross-correlation between two OFDM orthogonal chirp waveforms in a distributed-target scenario. The pulse length is $100 \mu \mathrm{s}$, and the chirp bandwidth is $100 \mathrm{MHz}$ at the center frequency of $5.2 \mathrm{GHz}$. In this simulation, it is assumed that 200 point scatterers exist within $1.5 \mathrm{~m} \times 3 \mathrm{~m}$ geometric resolution cell, thus simulating a distributed-target scenario. This figure shows the cross-correlation for a total of 1000 cells.

with the imaged swath. For example, if a pulse length of $100 \mu \mathrm{s}$ is transmitted, its echo signal length is approximately $430 \mu \mathrm{s}$ for $100-\mathrm{km}$ swath width at a $30^{\circ}$ incidence angle. In [30], an orthogonal waveform scheme also using a chirp signal, called short-term shift technique, has been introduced. It is emphasized that both OFDM and short-term-shift scheme require essentially the limitation of the receive signal length for the orthogonality. This induces the application of techniques to limit the delay "seen" on receive [34]. For this purpose, the present work uses DBF in elevation, thus implementing a spatial filter, as introduced in [29]. This can be understood as dividing the received signal into multiple subsets, where each subset is associated to a narrow receive beam, thus limiting the temporal delay of the corresponding received signal. In this way, the OFDM demodulation scheme can be applied to each subset while not sacrificing orthogonality. All details about the demodulation processing are presented in [29] and [35].

\section{Coherence}

A high spectral-temporal coherence is one of the most important design goals in the present MIMO SAR acquisition for both SAR interferometry (InSAR) and SAR polarimetry (PolSAR) applications. As aforementioned, the OFDM chirp scheme allows InSAR based on high coherence between SAR imageries reconstructed from different OFDM waveforms. This property is illustrated in Fig. 4 by evaluating the interferometric coherence between two SAR images of a real airborne SAR (E-SAR) image data. The E-SAR data parameters are summarized in Table I, and the simulation procedure is illustrated in Fig. 5. Two OFDM SAR raw data sets are reconstructed by the convolution between complex SAR image data $h(t)$ and two OFDM chirp waveforms, $s_{1}(t)$ and $s_{2}(t)$, which correspond to the second row in Fig. 1.

As shown in Fig. 4, the simulation result shows the quasiconstant coherence values within the OFDM operation range, whereas the coherence rapidly drops and converges to approximately 0.2 with the increase of the scene length. When a scene 


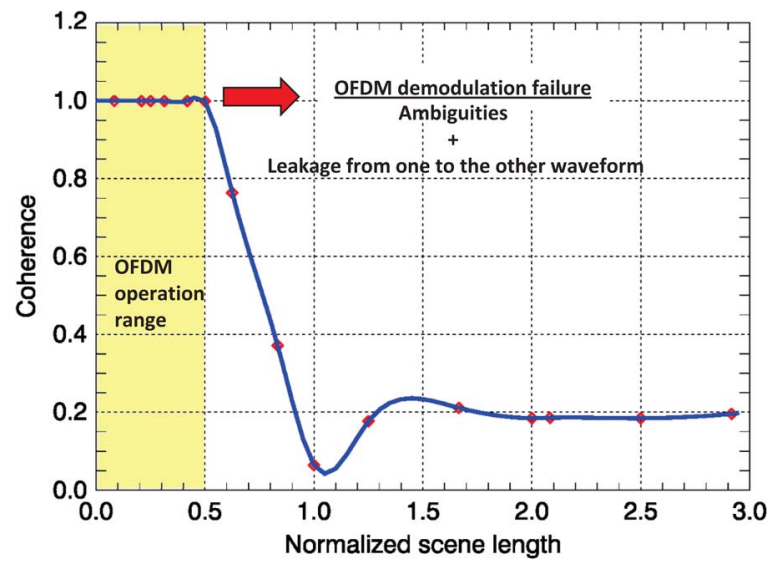

Fig. 4. Interferometric coherence for various target scene delay spread. The scene delay length is normalized by the OFDM pulse length $T$. The diamond symbols denote the simulation values, and the solid line is a curve fitting using spline interpolation.

TABLE I

PARAMETERS OF E-SAR DATA USED EQUiVALENT OFDM DATA GENERATION

\begin{tabular}{cc}
\hline Parameter & Value \\
\hline$f_{c}$ & $1.3 \mathrm{GHz}$ \\
polarization & $\mathrm{VV}$ \\
No of transmit antennas & 2 \\
PRF & $400 \mathrm{~Hz}$ \\
range samples & 1365 \\
bandwidth & $93 \mathrm{MHz}$ \\
chirp length $T_{p}$ & $5 \mu \mathrm{sec}$ \\
OFDM chirp length & $10 \mu \mathrm{sec}$ \\
sampling rate & $100 \mathrm{MHz}$ \\
azimuth samples & 2000 \\
\hline
\end{tabular}

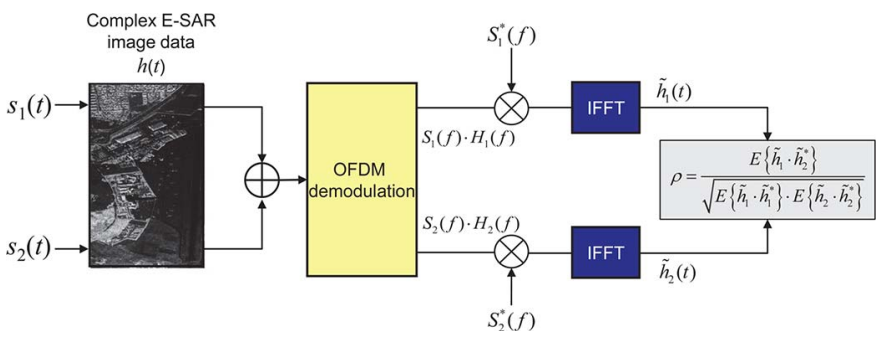

Fig. 5. Equivalent OFDM SAR image data generation from the original E-SAR image data for coherence evaluation.

length is shorter than a half an OFDM pulse, the OFDM signals are successfully demodulated, and the resulting image data spectra contain overlapping frequency components. However, as the scene length increases, the interpolation effect is reduced, and the OFDM demodulation also fails in the reconstruction of the image scene. Therefore, the coherence deteriorates strongly due to range ambiguities caused by OFDM demodulation as well as leakage from one to the other OFDM pulse.

To conclude, sufficient coherence for postprocessing can only be obtained for a shorter scene length than the OFDM pulse length; furthermore, the operation range of OFDM waveforms is bounded by the single chirp length $T_{p}$, which is used for the OFDM modulation. This conclusion corresponds to the basic idea behind traditional OFDM transmission that the information symbols are sent within a narrow coherence bandwidth, which is inversely proportional to the delay

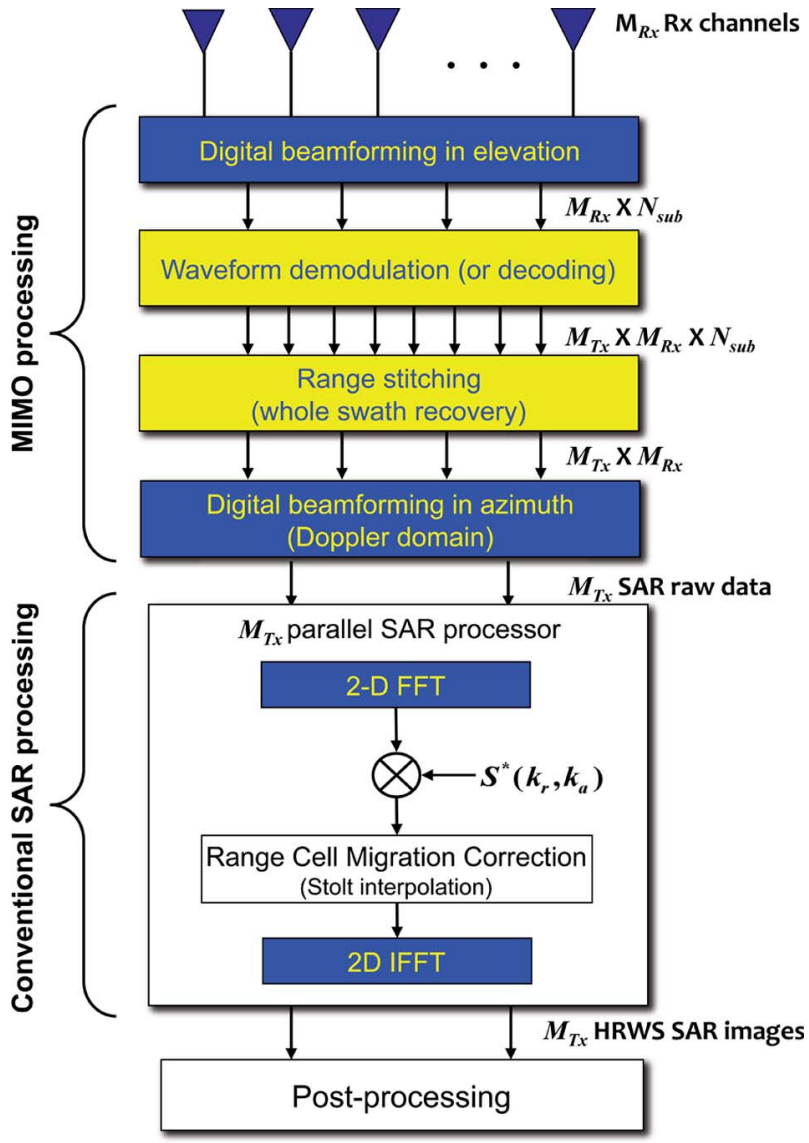

Fig. 6. Generic MIMO SAR processing schematic. Two major functions of the generic MIMO SAR processor are the MIMO signal processing and the conventional SAR processing. In this figure, the $\omega-k$ algorithm is considered as an example, so that the range cell migration correction is performed by the Stolt interpolation [37].

spread of channel, in order to avoid the frequency-selective fading [36].

\section{Generic MIMO SAR PRocessing}

Fig. 6 shows the schematic diagram of the generic MIMO SAR processor. Let the number of transmit antennas be $M_{\mathrm{Tx}}$, the number of receive antennas in azimuth be $M_{\mathrm{Rx}}$, and the number of subswaths be $N_{\text {sub }}$. The two primary objectives of the MIMO SAR processor are the successful separation of the multiple waveforms and their compression into HRWS SAR images. The DBF in elevation generates multiple narrow and high gain beams dividing the signal into subsets of short relative time delay. As detailed earlier, this is a necessity for separating the multiple simultaneously arriving echoes, which is referred to as demodulation in Fig. 6. This results in $M_{\mathrm{Tx}} \times M_{\mathrm{Rx}} \times$ $N_{\text {sub }}$ data streams, where each of the $N_{\text {sub }}$ demodulation output data streams corresponds to a narrow portion of the total swath; these are stitched together, leading to $M_{\mathrm{Tx}} \times M_{\mathrm{Rx}}$ channels. Azimuth reconstruction [13] is applied to eliminate the ambiguities in the individual channels, yielding the full Doppler data streams for each of the transmitted waveforms. These are then fed to the conventional SAR image processing.

Detailed processing of each step in Fig. 6 was introduced in previous publications [11], [29], [38], [39]. The OFDM 
demodulation scheme including the spatial filtering (DBF) is specifically explained in [29], and the other DBF techniques in azimuth are intensively investigated in [40]. As aforementioned, the increased number of phase centers in the MIMO configuration provides a great opportunity to merge two or more advanced multichannel modes into a single system. The basic idea behind the multimodal operation is to adjust the phase center distributions by reconfiguring the transmit antenna constellations for multiple modes. Based on the developed OFDM chirp waveforms, we present three examples of multimodal operation in Section V. The effect of the Doppler shift on the OFDM waveform is first analyzed in the following sections.

\section{DOPPLER EFFECT ON OFDM WAVEFORMS}

In this section, we investigate the feasibility of the OFDM waveform for the proposed MIMO SAR operations in terms of its orthogonal property in real spaceborne SAR scenario. The orthogonality between the subcarriers is fragile in complex propagation channels, due to a small spectral spacing. A typical example is the Doppler effect. In side-looking SAR, the Doppler effect results from the relative motion between sensor and targets and is exploited in SAR processing to achieve a high spatial resolution in azimuth. In contrast, the Doppler effect in fast-time domain is generally ignored in SAR processing since it is not recognizable within a SAR pulse length. To detect the Doppler shift, the pulse length should be at least one period of the Doppler frequency. This condition is generally not met in a pulse-based radar operation [41]. However, it is worthwhile investigating the Doppler effect in fast-time signals when the OFDM chirp length is increased, in order to extend subswath width. In this case, the Doppler effect becomes more significant. This section is dedicated to investigate the performance of the proposed OFDM waveform scheme regarding the Doppler shift and to derive a compensation algorithm to improve Doppler tolerance.

The Doppler shift may become a major cause of disturbance to the orthogonality between subcarriers. This can be mitigated by increasing the subcarrier spacing $\Delta f$; however, the reciprocal of the subcarrier spacing $\Delta f$ corresponds to the pulse length, which determines the subswath width. Therefore, the subcarrier spacing should be properly chosen. The intercarrier interference (ICI) is introduced to indicate the level of interference, i.e., loss of orthogonality, and to specify a boundary of the minimum subcarrier spacing. The ICI is the ratio between the single subcarrier power and the interference power contributed by all other subcarriers due to the Doppler shift.

To arrive at an expression for the ICI, let the total ICI power $P_{\mathrm{ICI}}$ be defined as the expected value of the Doppler distribution (see Appendix A)

$$
P_{\mathrm{ICI}} \triangleq \int_{-\infty}^{\infty}\left(1-\operatorname{sinc}^{2}\left(\pi \cdot f_{D} \cdot T\right)\right) \cdot p_{d}\left(f_{D}\right) \mathrm{d} f_{D}
$$

where $p_{d}\left(f_{D}\right)$ is the Doppler probability density function (pdf) and $\operatorname{sinc}(x)$ is defined as $\sin (x) / x$. In spaceborne SAR, the magnitude of the Doppler pdf is equivalent to the azimuth



Fig. 7. Estimated ICI power for a side-looking SAR scenario using the azimuth antenna pattern. The azimuth antenna pattern is assumed to be a sinc function $(\sin (x) / x)$.

antenna pattern limiting the Doppler spectrum to $\pm f_{D \text {,max }}$. Since the integral of a pdf is unity by definition, (5) can be simplified as follows:

$$
P_{\mathrm{ICI}} \approx 1-\int_{-f_{D, \max }}^{f_{D, \max }} p_{d}\left(f_{D}\right) \cdot \operatorname{sinc}^{2}\left(\pi \cdot f_{D} \cdot T\right) \mathrm{d} f_{D} .
$$

Applying a sinc-function shape antenna pattern as a Doppler pdf, we can directly estimate the total ICI power.

Fig. 7 shows the calculated ICI as a function of $f_{D} \cdot T$, which corresponds to the relative Doppler frequency to subcarrier spacing $\Delta f$ with $\Delta f=1 / T$. This plot is used to specify a lower bound of acceptable subcarrier spacing, with regard to the number of subcarriers. Assume a spaceborne SAR flying at $7 \mathrm{~km} / \mathrm{s}$ and using $85-\mathrm{MHz}$ signal bandwidth, 30- $\mu$ s pulse length, and $1.1 \times B$ sampling frequency. The subcarrier spacing $\Delta f$ of this system is $33 \mathrm{kHz}$. However, Fig. 7 shows that a threshold ICI of $-30 \mathrm{~dB}$ limits the maximum allowable Doppler frequency to $6 \%$ of a given subcarrier spacing. Consequently, the azimuth resolution is restricted by the desired ICI value. In this example, the maximum allowed Doppler frequency shift equals $2 \mathrm{kHz}(33 \mathrm{kHz} \times 0.06=2 \mathrm{kHz})$, for which the Doppler bandwidth of $4 \mathrm{kHz}$ gives a resolution of $1.75 \mathrm{~m}$ for a zero squint angle $(7 \mathrm{~km} / \mathrm{s} / 4 \mathrm{kHz}=1.75 \mathrm{~m})$. Fortunately, the Doppler frequency of a stationary target during transmission/reception is deterministic in spaceborne SAR due to stable satellite movement. A correction technique has been investigated and proposed in [35]. In case of a narrow subcarrier spacing, the instantaneous Doppler shift must be compensated prior to OFDM demodulation. It must be noted that these correction techniques assume a MIMO configuration without undersampling in azimuth and that any irregular Doppler shift (e.g., caused by moving objects) cannot be corrected by these methods.

\section{Multimodal Operation}

This section introduces possible MIMO SAR operations based on the OFDM chirp waveforms. It must be noted that 


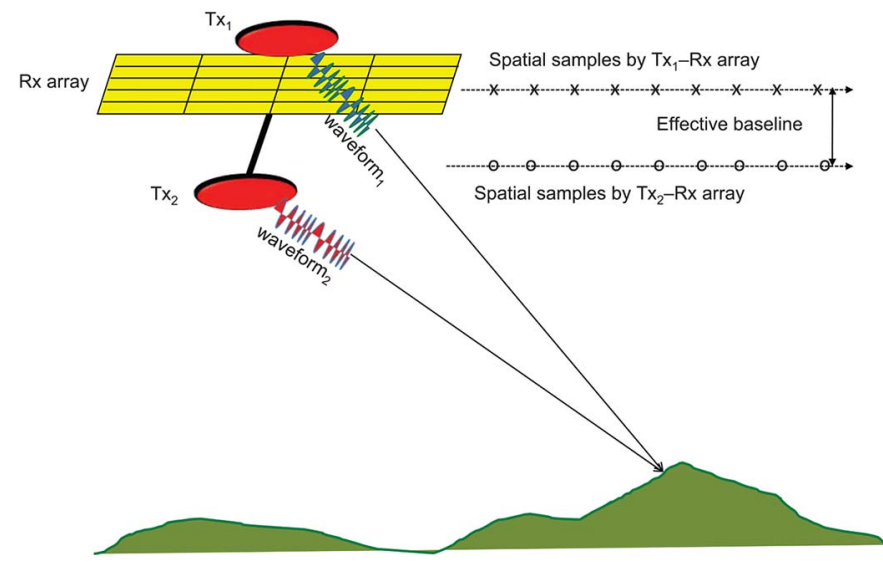

Fig. 8. Antenna constellation and the spatial sample distribution in the combination of the HRWS SAR imaging, and the across-track InSAR. The effective baseline is defined as the distance between spatial samples across track.

the MIMO antenna configurations in this section are using only two transmit antennas in order to present operation principles but are not limited to two transmit antennas at all.

\section{A. HRWS SAR Across-Track Interferometry}

The InSAR considered here is the across-track interferometry ${ }^{1}$ for topography measurement. The conventional implementation for InSAR mode is to deploy two receive antennas separated across track and to capture the pulse sent by a single transmit antenna. For HRWS SAR imaging, this imposes a constraint on satellite design due to a large second $\mathbf{R x}$ array needed. An alternative implementation is to employ two transmit antennas across track and a single receive antenna and to acquire data by transmission toggling. A recent study has explored the feasibility of InSAR satellite at Ka-band using two transmit antennas deployed using large booms across track [42]. However, this study considers transmission toggling, and its imaging performance is restricted by the fundamental SAR antenna constraint [2].

The present MIMO SAR also uses multiple transmit antennas instead of an additional large receive array and deploys it at a certain baseline across track. Applying the OFDM chirp waveforms, a simultaneous pulse transmission from transmit antennas becomes possible and overcomes the performance limitation. Fig. 8 shows the combination of the HRWS SAR imaging and the InSAR operation modes in the MIMO SAR, which is abbreviated here as HRWS InSAR mode. A simultaneous transmission of orthogonal OFDM pulses produces two separated phase center arrays denoted by the symbols " $x$ " and " $o$ " in Fig. 8, respectively. Each spatial sample array contributes to the HRWS SAR imaging mode independently, and thereby, two SAR images are reconstructed. In this case, a total of $M_{\mathrm{Rx}}$ receive and two transmit antennas are needed in the HRWS InSAR mode, whereas in the conventional approach, $M_{\mathrm{Rx}} \times 2$ receive and one transmit antennas are necessary to give out the same performance. Consequently, the presented MIMO

\footnotetext{
${ }^{1}$ For convenience, the term "InSAR" in this section means the single-pass across-track InSAR.
}

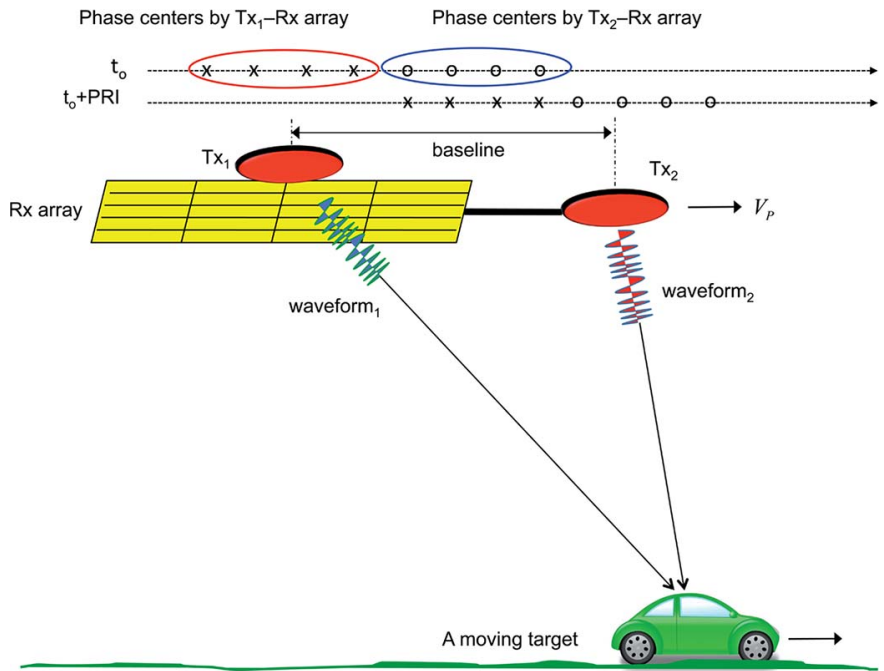

Fig. 9. Antenna constellation and the phase center distribution for GMTI. PRI stands for pulse repetition interval.

configuration is an attractive implementation for single-pass InSAR.

\section{B. HRWS SAR Imaging for GMTI}

When the second transmit antenna is arranged with a certain baseline along track, as shown in Fig. 9, $M_{\mathrm{Rx}}$ additional phase centers are produced between the second transmit antenna and the receive array. In other words, the number of phase centers can be increased by multiple transmit antennas at a low PRF, and they are used for along-track interferometry or GMTI without coverage reduction. The positions of the phase centers depend on the baseline. At the time instance $t_{o}$, both transmit antennas send pulses simultaneously, and thereby, two phase center arrays marked by " $x$ " and " $o$ " are formed along track. In the next transmission event, the other phase centers are formed consecutively. Thus, $2 M_{\mathrm{Rx}}$ phase centers along track are obtained by every single pulse transmission event. The increased number of phase centers improves GMTI performance and also expands potential ground coverage by reducing the required PRF. This operation mode can be very attractive for maritime surveillance and ocean surface current monitoring, which require a wide coverage. In GMTI, space time adaptive processing (STAP) technique is popularly applied to suppress ground clutter and to estimate the motion parameters of moving targets. More than two phase centers may improve the detection and estimation performance [43]. By employing more than two transmit antennas, the proposed MIMO SAR can easily increase the number of phase centers required for STAP performance enhancement. As a consequence, the MIMO SAR presents a great opportunity to improve both GMTI performance and coverage of SAR imaging simultaneously.

\section{HRWS PolSAR and Pol-InSAR}

In the conventional PolSAR mode, each vertically and horizontally polarized signal is radiated by an alternative transmission mode, and two scattering components are acquired by 


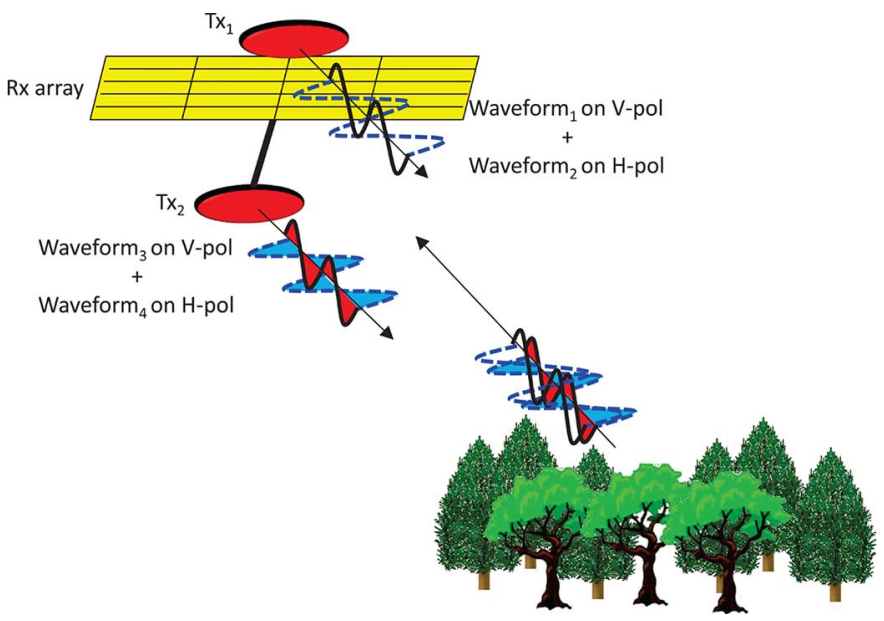

Fig. 10. Fully polarimetric data acquisition based on orthogonal waveforms combined with across-track interferometric data acquisition. Dual-polarization antennas with a good cross-polarization level are the premise of the HRWS Pol-InSAR mode.

each transmission event. Hence, a full scattering matrix can be obtained through the interleaved data acquisition at the cost of a higher PRF. In this traditional mode, the HRWS SAR also retains this restriction for PolSAR mode. In the MIMO SAR, orthogonal waveforms assigned to each polarization allow a simultaneous acquisition of fully polarimetric data [44], and thereby, the present system overcomes the restriction of the conventional PolSAR data acquisition. Furthermore, the improvement in PolSAR mode can be extended to polarimetric InSAR (Pol-InSAR) mode, as shown in Fig. 10.

The orthogonal waveforms are radiated through both polarization channels of each transmit antenna $\mathrm{Tx}_{1}$ and $\mathrm{Tx}_{2}$ in Fig. 10. For the HRWS Pol-InSAR mode, a total of four orthogonal waveforms are required for vertical and horizontal polarizations of both transmit antennas

$$
\mathbf{S}=\left[\begin{array}{llll}
S_{1} & S_{2} & S_{3} & S_{4}
\end{array}\right]^{\mathrm{T}}
$$

To put it concretely, waveforms $S_{1}$ and $S_{2}$ are transmitted with $\mathrm{V}$ and $\mathrm{H}$ polarizations by the $\mathrm{Tx}_{1}$ antenna, respectively, and waveforms $S_{3}$ and $S_{4}$ are assigned likewise for the $\mathrm{Tx}_{2}$ antenna. Depending on the target properties, the polarization state of the transmitted signals is transformed, and two orthogonally polarized signals are delivered to a receive antenna from each waveform. The polarization channel transfer function $\mathbf{H}_{1}$ for the $\mathrm{Tx}_{1}$ and receive antennas is given as

$$
\mathbf{H}_{1}=\left[\begin{array}{ll}
H_{1, v v} & H_{1, v h} \\
H_{1, h v} & H_{1, h h}
\end{array}\right]
$$

and in the same manner, $\mathbf{H}_{2}$ for the $\mathrm{Tx}_{2}$ and receive antennas is described as

$$
\mathbf{H}_{2}=\left[\begin{array}{ll}
H_{2, v v} & H_{2, v h} \\
H_{2, h v} & H_{2, h h}
\end{array}\right]
$$

where the subscripts $v v, v h, h v$, and $h h$ indicate the polarization responses of the received signals. The fully polarimetric

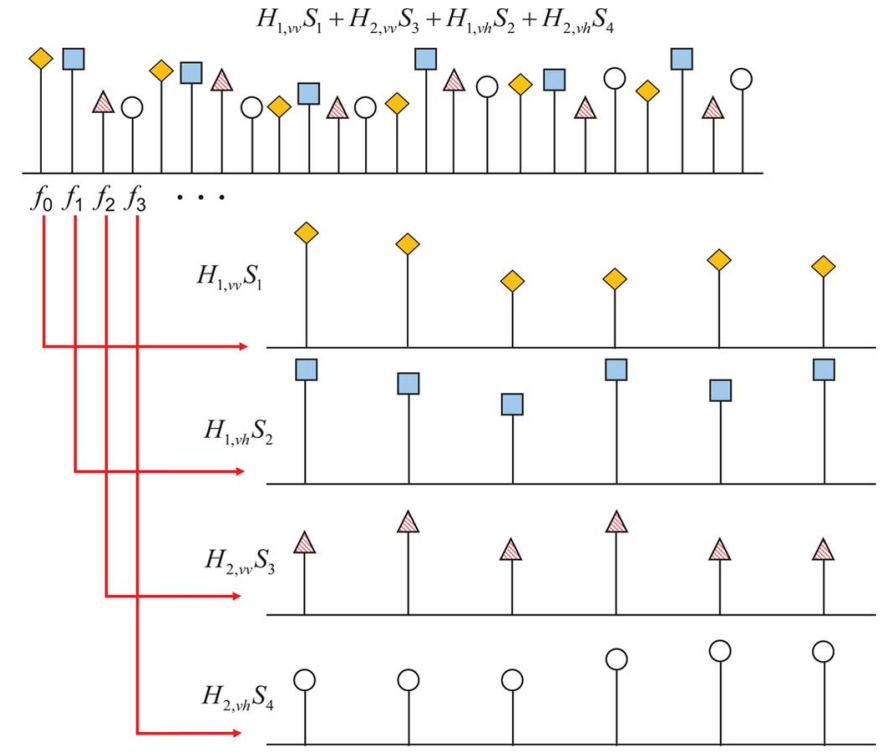

Fig. 11. Example signal spectrum captured by the V-polarization channel of a single receive antenna. All polarimetric information is preserved and separable by the polyphase decomposition.

channel matrix is

$$
\mathbf{H}=\left[\begin{array}{ll}
\mathbf{H}_{1} & \mathbf{H}_{2}
\end{array}\right]
$$

Therefore, the signals captured by a single receive antenna $\mathbf{R}$ are described as

$$
\begin{aligned}
\mathbf{R} & =\left[R_{v} R_{\mathrm{h}}\right]^{\mathrm{T}}=\mathbf{H} \cdot \mathbf{S} \\
& =\left[\begin{array}{l}
H_{1, v v} S_{1}+H_{2, v v} S_{3}+H_{1, v h} S_{2}+H_{2, v h} S_{4} \\
H_{1, h v} S_{1}+H_{2, h v} S_{3}+H_{1, h h} S_{2}+H_{2, h h} S_{4}
\end{array}\right]
\end{aligned}
$$

where $R_{v}$ and $R_{h}$ denote the captured signals by the $\mathrm{V}$ - and $\mathrm{H}$ polarization channels of the receive antenna, respectively. As a result, the fully polarimetric data are acquired by a single transmission event. The vertically and horizontally polarized signals are first separated by the antenna, and then, the polarization component carried by each orthogonal waveform is retrieved by the waveform demodulation. Fig. 11 illustrates the polarization retrieval by the polyphase decomposition during the OFDM demodulation.

\section{ExAmple System Design}

In this section, we design an example MIMO SAR system based on the OFDM chirp waveform. Aside from the antenna parameters, other system and geometric parameters are chosen according to traditional rules, such as the radar equation or the parameters of existing systems. As introduced in the previous section, the transmit antenna constellation is reconfigurable, depending on the operation mode. The baseline between the transmit antennas is defined by the specific application and is not fixed in the following analysis.

Table II lists the designed system and geometric parameters. The pulse duration of $150 \mu$ s leads to the duty cycle of $24.1 \%$ for the highest PRF of $1.61 \mathrm{kHz}$. This value can be reduced by employing a shorter OFDM pulse at the cost of increasing 
TABLE II

SYSTEM PARAMETERS OF THE EXAMPLE DESIGN

\begin{tabular}{|c|c|c|}
\hline \multicolumn{2}{|c|}{ Parameters } & Values \\
\hline Geometry & $\begin{array}{l}\text { Swath width } \\
\text { No. of subswaths } \\
\text { orbit height } \\
\text { velocity } \\
\text { spatial resolution } \\
\text { center frequency }\end{array}$ & $\begin{array}{c}\sim 100 \mathrm{~km} \\
14 \\
560 \mathrm{~km} \\
7560 \mathrm{~m} / \mathrm{sec} \\
1 \mathrm{~m} \times 1.5 \mathrm{~m} \\
\text { (elevation } \times \\
\text { azimuth) } \\
9.65 \mathrm{GHz}\end{array}$ \\
\hline Transmit antenna & $\begin{array}{l}\text { length } \\
\text { height } \\
\text { gain } \\
\text { No. of antennas }\end{array}$ & $\begin{array}{c}2.5 \mathrm{~m} \\
0.25 \mathrm{~m} \\
39 \mathrm{dBi} \\
2\end{array}$ \\
\hline Receive array & $\begin{array}{l}\text { total length } \\
\text { total height } \\
\text { gain } \\
\text { subarray length } \\
\text { subarray height } \\
\text { radiator per subarray } \\
\text { radiator height } \\
\text { azimuth panel } \\
\text { elements in elevation }\end{array}$ & $\begin{array}{c}9.6 \mathrm{~m} \\
3.5 \mathrm{~m} \\
56 \mathrm{dBi} \\
1.6 \mathrm{~m} \\
0.083 \mathrm{~m} \\
3 \\
0.0276 \mathrm{~m} \\
6 \\
42\end{array}$ \\
\hline $\mathrm{H} / \mathrm{W}$ system & $\begin{array}{c}\text { peak power } \\
\text { average power } \\
\text { bandwidth } \\
\text { pulse duration } \\
\text { duty cycle } \\
\text { noise figure } \\
\text { loss } \\
\text { sampling frequency } \\
\text { pulse subcarrier spacing } \\
\text { No. of pulse subcarriers }\end{array}$ & $\begin{array}{c}5 \mathrm{~kW} \\
\max .1 .208 \mathrm{~kW} \\
250 \mathrm{MHz} \\
150 \mu \mathrm{sec} \\
24.1 \% \\
3.75 \mathrm{~dB} \\
3 \mathrm{~dB} \\
275 \mathrm{MHz} \\
6.67 \mathrm{kHz} \\
4.125 \times 10^{4}\end{array}$ \\
\hline
\end{tabular}

the receive array height. In this system operation, a severe nonuniform sampling will not occur due to the narrow PRF range. In the case that a wide range of PRF is considered, the degree of nonuniform sampling will be significant and reach the maximum at the highest PRF. In the designed system, it is assumed that all receive channels have the identical noise figure of $3.75 \mathrm{~dB}$ and a system loss of $3 \mathrm{~dB}$. The required peak and average power of this system are higher than those of the TerraSAR-X system (2-kW peak) in order to achieve the desired SNR over the $100-\mathrm{km}$ swath width. As a rule, the sampling frequency of $275 \mathrm{MHz}$ includes a $10 \%$ guard band, and then, the pulse length of $150 \mu$ s leads to 41250 subcarriers with the $6.67-\mathrm{kHz}$ subcarrier spacing. In this example, we assume that the ICI due to instantaneous Doppler shift is compensated by the method introduced in [35]. Regarding the undersampling for HRWS SAR imaging in the MIMO SAR, the reconstruction algorithm [13] recovers the original Doppler spectrum prior to the Doppler compensation. This approach has been used for frequency-modulated continuous-wave (FMCW) SAR with DBF in [45] and also for the very high resolution SAR data processing [46]. Therefore, the ICI issue is not included in the performance estimation in Section VI-A. To improve the computation speed of DFT/inverse DFT, one can select a number of subcarriers that are equal to a power of two. This MIMO SAR antenna is composed of six panels in azimuth and 42 receive subarrays in elevation in each panel. The receive subarray consists of three $\mathrm{X}$-band radiators in elevation and azimuth, respectively. Fig. 12 depicts the MIMO SAR antenna configuration and geometric parameters in this example design.

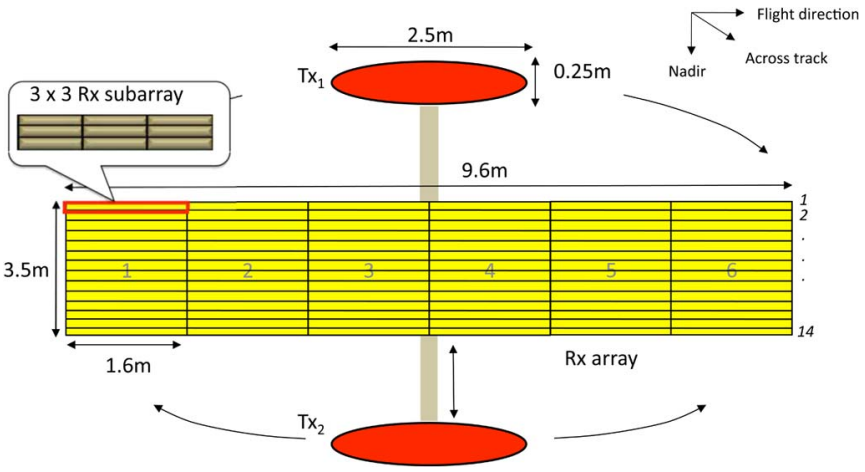

Fig. 12. MIMO SAR antenna configuration of the example design.

This example system was designed for the multimodal operation, based on the developed techniques. If the designed MIMO SAR system is dedicated to the HRWS SAR imaging operation mode, the multiple transmit antennas can be used to reduce the total antenna size as well. The total area of the six receive subarrays and two transmit antennas in this design is $35 \mathrm{~m}^{2}$. By arranging the second transmit antenna along track at a proper distance, only three receive subarrays are required for the same HRWS SAR imaging performance of the original design, instead of the six subarrays, according to the coarray concept [47]. In this case, the total antenna area is reduced to $18.14 \mathrm{~m}^{2}$, which is $51.76 \%$ smaller than the original design.

\section{A. Performance Estimation}

This section is dedicated to the evaluation of the performance of the designed MIMO SAR system, in terms of the system sensitivity and the range ambiguity.

1) NESZ: The noise equivalent sigma zero (NESZ) is a parameter indicating the system radiometric sensitivity, which is defined as the backscattering coefficient corresponding to an SNR that is equal to unity [48]. The bistatic configuration of the MIMO SAR leads to different antenna gains in the transmission and reception, meaning that the NESZ is formulated for these gains as follows:

$$
\mathrm{NESZ}=\frac{256 \cdot\left(\pi \cdot R_{o}\right)^{3} \cdot k_{\mathrm{bol}} \cdot T_{\mathrm{sys}} \cdot L_{\mathrm{ant}} \cdot v \cdot B \cdot \sin \theta}{P_{t} \cdot 2 T_{p} \cdot \mathrm{PRF} \cdot G_{T}(\theta) \cdot G_{R}(\theta) \cdot \lambda^{3} \cdot c_{o}}
$$

where $T_{\text {sys }}$ is the equivalent system noise temperature, $c_{o}$ is the speed of light, $k_{\mathrm{bol}}$ is the Boltzmann's constant of $1.3807 \times$ $10^{-23} \mathrm{~J} \cdot K^{-1}, P_{t}$ is the peak transmit power, $L_{\text {ant }}$ is the loss of antenna feed networks, $G_{T}$ and $G_{R}$ are the gain patterns of the transmit antenna and the receive array, respectively, $\theta$ denotes the elevation angle, and $\lambda$ is the wavelength. Fig. 13 shows the estimated NESZ values for the designed $2 \times 6$ MIMO SAR system (cf. Fig. 12).

The calculated NESZ of each subswath is indicated by the dashed-dotted line. There is approximately 5 - $\mathrm{dB}$ difference between the best and the worst values within the whole swath. In the designed system, 14 beams, i.e., subswaths, cover the whole swath. It should be emphasized that the NESZ was separately calculated for each transmit antenna. In other words, adding a transmitter for sending an orthogonal signal does not improve SNR. For the multimodal operation, this system 


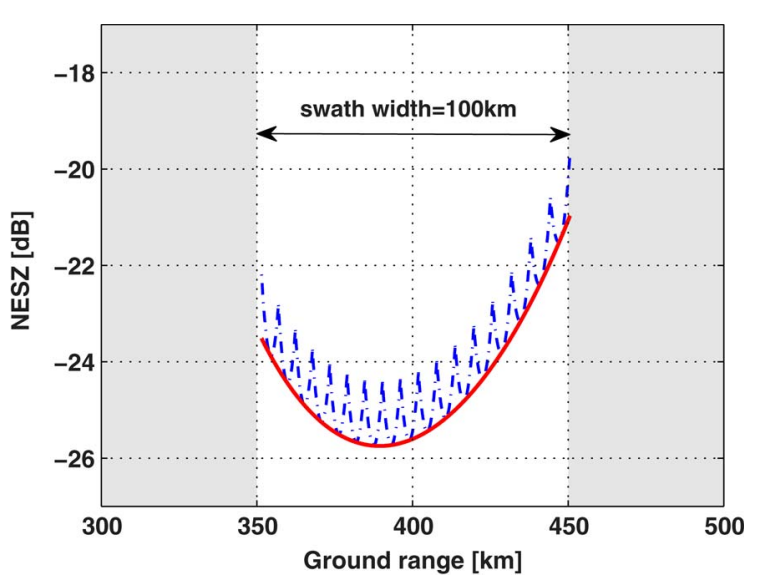

Fig. 13. NESZ of the example system. The dashed-dotted line denotes the NESZ of each subswath, and the solid line highlights the coherent combination of the subswath data. The system is designed to achieve NESZ $<-20 \mathrm{~dB}$, which leads to a satisfactory SNR value for most applications. The antenna elevation pattern was tapered by a Dolph-Chebyshev window for a sidelobe level of $-35 \mathrm{~dB}$.

reconstructs multiple images for the interferometry or the polarimetry, separately. The multiple transmit antennas can be used for the NESZ improvement as well, as long as the multiple images are coherently summed after the coregistration.

2) RASR: Range ambiguities are caused by overlapping echoes from preceding and succeeding pulses to desired signals [32]. The antenna sidelobes have a decisive influence on the range ambiguity level. The range-ambiguity-to-signal ratio (RASR) is used to indicate the range ambiguity effect in SAR images with regard to the antenna pattern and the OFDM demodulation process. For the MIMO SAR system, this parameter is defined as follows:

$$
\begin{array}{r}
\text { RASR }=\underbrace{\frac{R_{o}^{3} \cdot \sin \left(\theta_{\text {inc }}\right)}{\left|C_{2 \text { way }}(\theta)\right|^{2}} \cdot \sum_{\substack{m \neq 0 \\
m=-N_{n}}}^{N_{f}} \frac{\left|C_{2 \text { way }}\left(\theta_{m}\right)\right|^{2}}{R_{o, m}^{3} \cdot \sin \left(\theta_{\text {inc }, m}\right)}}_{\operatorname{RASR}_{\text {con }}} \\
+\underbrace{\frac{R_{o}^{3} \cdot \sin \left(\theta_{\text {inc }}\right)}{\left|C_{2 \text { way }}(\theta)\right|^{2}} \cdot \frac{\left|C_{2 \text { way }}(\theta+\Delta \theta)\right|^{2}}{R_{o}^{3} \cdot \sin \left(\theta_{\text {inc }}+\Delta \theta_{\text {inc }}\right)}}_{\text {RASR }_{\text {ofdm }}}
\end{array}
$$

where $\theta_{\text {inc }}$ denotes the incidence angle and $C_{2 \text { way }}$ is the twoway antenna pattern. The subscript $m$ indicates the ambiguous signal order. $N_{n}$ and $N_{f}$ give the number of pulses considered in the calculation in both near and far ranges, respectively. $R_{o}$ is the original distance to a target, and $R_{o, m}$ is the slant range between the radar sensor and the position of the $m$ th ambiguous signal source, corresponding to an elevation angle $\theta_{m}$ and incidence angle $\theta_{\mathrm{inc}, m} . \Delta \theta$ and $\Delta \theta_{\mathrm{inc}}$ are the elevation look angle and incidence angle separations corresponding to $T$, respectively. Equation (13) is composed of two parts. The first stands for the conventional RASR value, denoted by RASR $\mathrm{Ron}_{\text {, }}$, and the second represents the additional value $\mathrm{RASR}_{\text {ofdm }}$, caused by the circular-shift addition process in the OFDM demodulation, which are $T_{p}$ apart from the original position in time domain. In other words, the RASR ${ }_{\text {ofdm }}$ indicates the interference level from adjacent subswaths, since the circularly

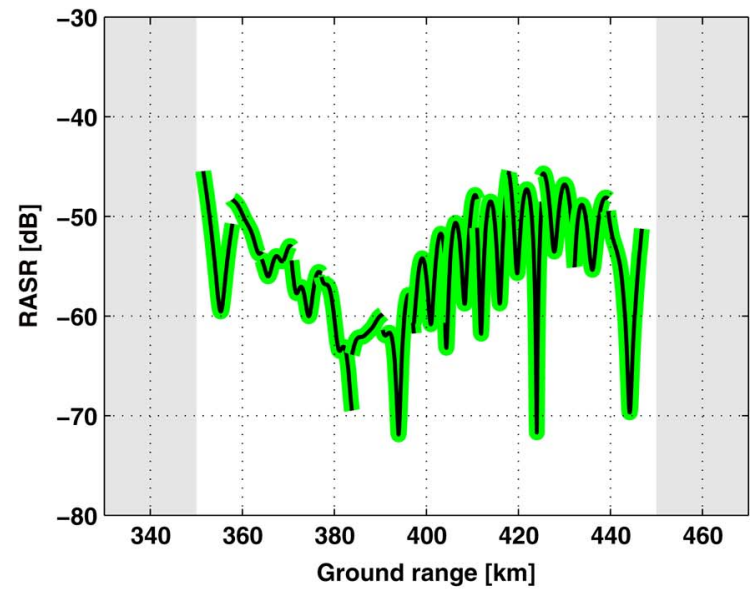

(a)

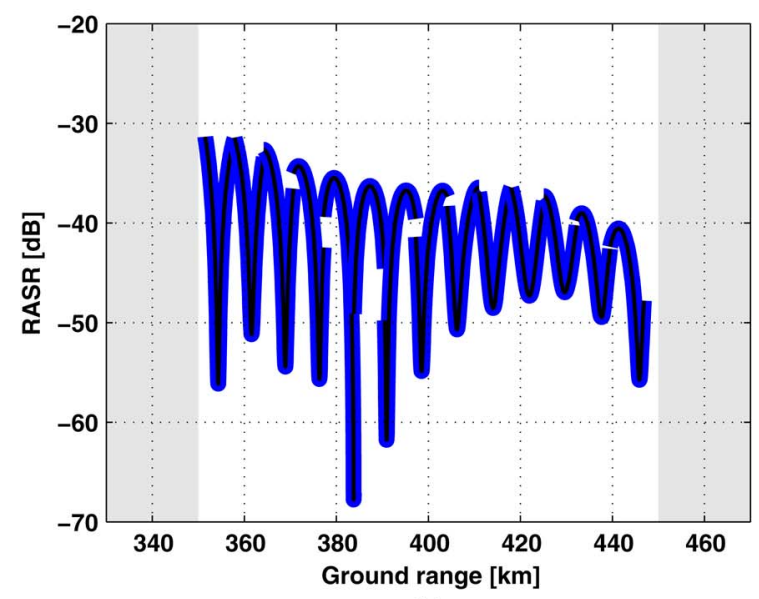

(b)

Fig. 14. Computed RASR over the whole swath: (a) Conventional RASR part and (b) the final RASR containing the OFDM contribution mainly caused by the circular-shift addition during the OFDM demodulation (cf. [29]). The receive antenna elevation pattern was tapered by a Dolph-Chebyshev window for a sidelobe level of $-35 \mathrm{~dB}$.

shifted signal originates from the succeeding subswath region. This interference rises up due to imperfect spatial filtering (mainly due to sidelobes) by DBF on receive.

Fig. 14(a) shows the conventional RASR term in (13). The range ambiguities are suppressed in the antenna pattern by $45 \mathrm{~dB}$. The narrow elevation beam of the receive array reduces the echo spread of each subswath. Furthermore, the reduced PRF via the multiple receive subarrays in azimuth results in an increase of the angular separation between the desired signal and the range ambiguity signals. However, the final RASR is increased, as shown in Fig. 14(b), since the range ambiguity caused by the circular-shift addition is added. This result implies that the spatial filtering performance is critical. In this design, we suppress the sidelobes in the receive antenna pattern up to $-35 \mathrm{~dB}$ by the Dolph-Chebyshev taper and achieve the RASR better than $-30 \mathrm{~dB}$.

\section{B. Performance Comparison}

The performance of the MIMO SAR system is compared with that of TerraSAR-X in terms of several geometric and radiometric parameters. These performance parameters are listed 
This article has been accepted for inclusion in a future issue of this journal. Content is final as presented, with the exception of pagination.

TABLE III

Characteristic Parameters OF TERRASAR-X AND MIMO SAR

\begin{tabular}{|c|c|c|c|c|c|c|}
\hline System & Mode & Polarization & Swath & $\begin{array}{l}\text { Azimuth } \\
\text { resolution }\end{array}$ & NESZ & RASR \\
\hline \multirow{3}{*}{ TerraSAR-X } & Stripmap & $\begin{array}{l}\text { Single } \\
\text { Dual }\end{array}$ & $\begin{array}{l}30 \mathrm{~km} \\
15 \mathrm{~km}\end{array}$ & $\begin{array}{l}3.3 \mathrm{~m} \\
6.6 \mathrm{~m}\end{array}$ & \multirow{3}{*}{$-19 \mathrm{~dB}$} & \multirow{3}{*}{$<-17 \mathrm{~dB}$} \\
\hline & Spotlight & $\begin{array}{c}\text { Single } \\
\text { Dual }\end{array}$ & $\begin{array}{l}10 \mathrm{~km} \\
10 \mathrm{~km}\end{array}$ & $\begin{array}{l}1.7 \mathrm{~m} \\
3.4 \mathrm{~m}\end{array}$ & & \\
\hline & ScanSAR & Single & $100 \mathrm{~km}$ & $18.5 \mathrm{~m}$ & & \\
\hline \multirow{3}{*}{ MIMO SAR } & HRWS PolSAR & Quad & $100 \mathrm{~km}$ & $1.5 \mathrm{~m}$ & \multirow{3}{*}{$-20 \mathrm{~dB}$} & \multirow{3}{*}{$<-30 \mathrm{~dB}$} \\
\hline & HRWS InSAR & Single & $100 \mathrm{~km}$ & $1.5 \mathrm{~m}$ & & \\
\hline & HRWS GMTI & Single & $66 \mathrm{~km}$ & $1.5 \mathrm{~m}$ & & \\
\hline
\end{tabular}

in Table III. In stripmap mode, the TerraSAR-X system produces SAR images with 3.3-m azimuth resolution over 30-km swath in single polarization mode. The azimuth resolution is improved in spotlight mode to $1.7 \mathrm{~m}$ at the cost of ground coverage $(10 \mathrm{~km})$. In ScanSAR mode, the ground coverage is increased by steering the antenna beam across track, but it allows only the azimuth resolution of $18.5 \mathrm{~m}$ [49]. This is the fundamental restriction of conventional SAR systems. This antenna constraint problem restricts the system performance even more in the polarimetric data acquisition. Due to toggling of the polarization between consecutive pulses on transmit, a higher PRF value is required to preserve the resolution, and it yields the reduction of the swath width or vice versa. This tradeoff is clearly seen in stripmap and spotlight modes in Table III for the dual-polarization mode.

In contrast, the designed MIMO SAR system shows steady imaging performance independent of the operation modes. As described in Section V, the MIMO SAR system does not require a higher PRF value in the polarimetric and interferometric modes, owing to the simultaneous transmission of OFDM waveforms. As a consequence, the performance improvement by the MIMO SAR is considerable compared to the conventional single-channel system. A remark should be given to the HRWS GMTI mode in Table III. For the parameter estimation of moving targets, at least three azimuth channels are needed. In this design example, each of the four phase centers is used to build an HRWS SAR image from the antenna constellation for the HRWS GMTI (cf. Fig. 9). This requires a PRF increase of up to $2.415 \mathrm{kHz}$, and therefore, the swath width is reduced in comparison with the other modes. From this example design, it becomes clear that the MIMO SAR allows a substantial performance enhancement for the polarimetric and interferometric modes, and the OFDM waveform scheme plays a key role in the MIMO SAR multimodal operation.

\section{CONCLUSION}

In this paper, we have presented a novel spaceborne MIMO SAR concept based on the OFDM waveform and DBF techniques. The proposed MIMO SAR aims at the multimodal operation, overcoming the inherent limitations of the stateof-the-art SAR system. It is emphasized that the DBF and the OFDM waveform scheme are the predominant tools to implement the multimodal operation. In particular, the OFDM waveform scheme allows the simultaneous transmission of multiple orthogonal pulses and also provides sufficient coher- ence between reconstructed multichannel SAR images for interferometric and polarimetric applications. This unique feature of the OFDM waveform makes it possible to effectively use the MIMO SAR system. As an example, we extended a digital receive array system (HRWS SAR) by adding an extra transmit antenna in order to increase the spatial degree of freedom. By reconfiguring the transmit antenna constellation, the MIMO SAR system composes additional phase centers along track or across track and can satisfy the needs of the HRWS imaging and other multichannel data acquisition modes at the same time. The designed MIMO SAR system showed the steady geometric and radiometric performance, while the performance of a single-channel system depends on operation modes due to the fundamental SAR antenna constraint.

\section{Future Topics}

An important topic that should be further investigated is the performance validation for the random scattering behavior of natural scene [29], [35], [44]. Experimental studies using a ground-based or airborne system are expected to provide a full understanding of the presented OFDM signal responses for a general imaging scenario with distributed targets. Moreover, from a technical viewpoint, the present work also inspires further ideas to accelerate technical progress in the field of MIMO SAR. One of the important issues for future activities is the combination of MIMO SAR with a large reflector antenna. When combined with a digital feed array, the reflector antenna system allows various advanced modes with the aid of DBF [50]. This high potential has been intensively studied for the Tandem-L mission, which is a proposal for an innovative interferometric L-band radar mission [51]. In particular, the capability of multibeam generation of the reflector antenna system is of great interest for MIMO SAR due to its excellent spatial filtering performance. A further point involves bistatic and multistatic SAR systems. In this case, individual transmit and receive antennas are carried by separate satellites, which can form multiple baselines for various interferometric applications [52]. Furthermore, this configuration offers an opportunity of using continuous signals like FMCW. This type of signal allows one to reduce the high power demands on transmit and to simplify system hardware [6]. By generating continuous OFDM waveforms, the multistatic system will be effectively combined with the MIMO SAR operation modes. A dual use of OFDM signals for communication and radar remote sensing is also a great interest that should be pursued. Its high 
potential has been intensively investigated for short-range radar applications [53], [54]. For spaceborne SAR applications, communication satellites sending OFDM signals may be used as transmitters of opportunity in geostationary orbit, and multiple small receiver-only satellites acquire data in low Earth orbit. There are many other topics, like sparse distributed arrays of satellites, that should be further studied in the scope of future activities. We hope that this paper will inspire diverse ideas and boost many collaborative efforts for substantial progress in spaceborne MIMO SAR technology.

\section{APPENDIX A}

\section{DERIVATION OF ICI}

In this appendix, (5) is derived in detail. The ICI is used to specify a boundary of the minimum subcarrier spacing for given channels. Doppler shift results in interferences between subcarriers and amplitude attenuation of the subcarrier. Since the Doppler shift is not described by a single value due to multipath signals, the Doppler spectrum model is commonly used in the ICI estimation.

Assume that $s[n]$ is an OFDM chirp signal for twotransmitter case in discrete time, and its Fourier transform is $S[p]$. When $s[n]$ is transmitted and returns to a sensor, the received signal $r[n]$ is described as follows:

$$
\begin{array}{r}
r[n]=\frac{1}{2 N} \sum_{p=0}^{2 N-1} S[p] \cdot \exp \left(j \frac{2 \pi}{2 N}(n-k)(p+\Delta p)\right) \\
\cdot \operatorname{rect}\left[\frac{n-k}{2 N}\right]
\end{array}
$$

where $n$ and $p$ are the discrete time and frequency index, respectively, $N$ is the total time samples of a single chirp, $k$ is the delay, and $\Delta p$ represents the Doppler shift of the $p$ th subcarrier. Note that the signal amplitude and antenna pattern ${ }^{2}$ are omitted in this derivation for simplicity. The signal is demodulated by the $2 N$-point DFT on receive. As in the prior section, the demodulated signal is derived as

$$
\begin{aligned}
\mathcal{F}\{r[n]\} & =R[p]=\sum_{n=0}^{2 N-1} r[n] \cdot \exp \left(-j \frac{2 \pi}{2 N} n p\right) \\
& =S[p] \cdot \exp \left(-j \frac{2 \pi}{2 N} k(p+\Delta p)\right) \cdot \operatorname{sinc}(p+\Delta p) .
\end{aligned}
$$

In the following, this signal spectrum with the Doppler shift is reformulated in terms of a specific subcarrier and the rest. Fig. 15 depicts the principle of the following signal model. The received signal spectrum is decomposed into the discrete frequency domain $p$ and the subcarrier domain $q$. Thereby, one can describe the interferences from the sidelobes of subcarrier functions $\left(q \neq q_{o}\right)$ to the reference subcarrier $q_{o}$. Of course, if

\footnotetext{
${ }^{2}$ The antenna pattern is reflected to a Doppler spectrum model for a sidelooking SAR geometry.
}
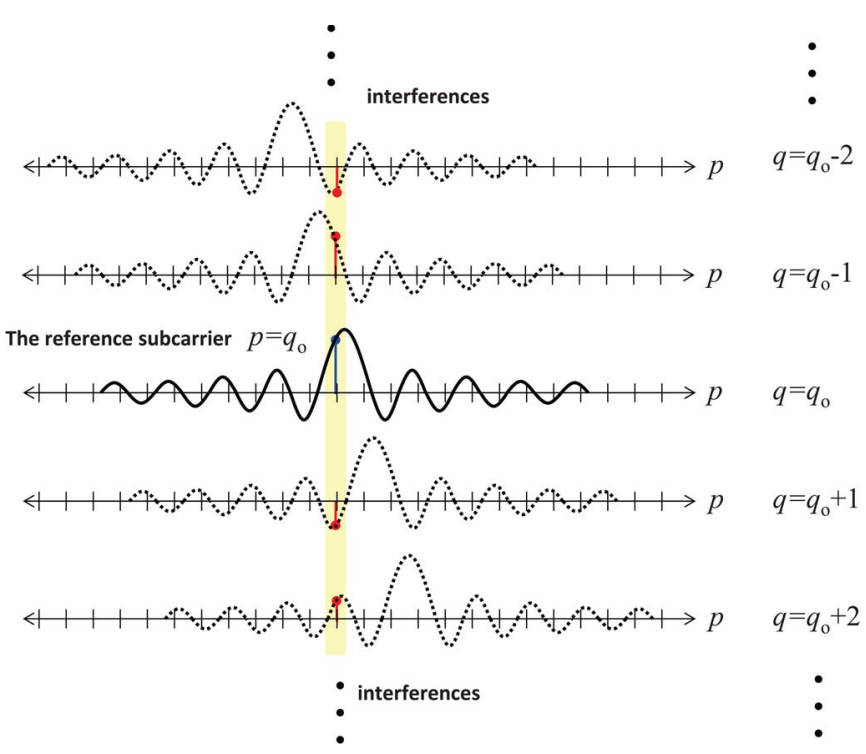

Fig. 15. ICI contributions of other subcarriers $\left(q \neq q_{o}\right)$ to the reference subcarrier $q_{o}$.

there is no Doppler shift, the sidelobes of all subcarrier functions correspond to the nulls. Put simply, they are orthogonal.

To derive the interferences on the $q_{o}$ subcarrier, (15) is described again as

$$
\begin{aligned}
R[p]= & S[p] \cdot \operatorname{sinc}\left(p-q_{o}+\Delta q_{o}\right) \cdot \exp \left(-j \frac{2 \pi}{2 N} k\left(p-q_{o}+\Delta q_{o}\right)\right) \\
& +\sum_{\substack{q=0 \\
q \neq q_{o}}}^{2 N-1} S[p] \cdot \operatorname{sinc}(p-q+\Delta q) \cdot \exp \left(-j \frac{2 \pi}{2 N} k(p-q+\Delta q)\right)
\end{aligned}
$$

where $\Delta q_{o}$ and $\Delta q$ denote the Doppler shifts of the reference $q_{o}$ th subcarrier and the other subcarriers, respectively. Equation (16) consists of two terms: The first term describes the $q_{o}$ subcarrier with the Doppler shift, and the second term presents the ICI contribution of the others to the $q_{o}$ subcarrier. For convenience, (16) is rewritten in a continuous signal form, according to the relationship $2 \pi / N T_{s}=2 \pi \Delta f$ and $T_{p}=2 N T_{s}$, as follows:

$$
\begin{aligned}
R\left(f_{p}\right)= & S\left(f_{p}\right) \cdot \operatorname{sinc}\left(2\left(f_{p}-f_{q_{o}}+f_{d}^{q_{o}}\right) T_{p}\right) \\
& \cdot \exp \left(-j 2 \pi\left(f_{p}-f_{q_{o}}+f_{d}^{q_{o}}\right) k \cdot T_{s}\right) \\
& +\sum_{\substack{q=0 \\
q \neq q_{o}}}^{2 N-1} S\left(f_{p}\right) \cdot \operatorname{sinc}\left(2\left(f_{p}-f_{q}+f_{d}^{q}\right) T_{p}\right) \\
& \cdot \exp \left(-j 2 \pi\left(f_{p}-f_{q}+f_{d}^{q}\right) k T_{s}\right)
\end{aligned}
$$

where $f_{q}=2 \pi \cdot q \cdot \Delta f$ and $f_{d}^{q}=2 \pi \cdot \Delta q \cdot \Delta f$. In the same way, $f_{p}=2 \pi \cdot p \cdot \Delta f$. The interest is the power level of interference. The exponential terms in (17) have no effect on the ICI due to the amplitude of unity, and $\left|S\left(f_{p}\right)\right|$ is also assumed to be unity. Therefore, these can be omitted in further power 
calculations. The spectral power at $f_{q_{o}}$ (i.e., $p=q_{o}$ ) is a sum of the reference subcarrier power and the ICI power

$$
\begin{aligned}
\left|R\left(f_{q_{o}}\right)\right|^{2}= & \underbrace{\operatorname{sinc}^{2}\left(2 \cdot f_{d}^{q_{o}} \cdot T_{P}\right)}_{\text {reference }} \\
& +\underbrace{\sum_{\substack{q=0 \\
q \neq q_{o}}}^{2 N-1} \operatorname{sinc}^{2}\left(2\left(f_{q_{o}}-f_{q}+f_{d}^{q}\right) \cdot T_{p}\right)}_{\text {ICI }} \\
= & \left|R_{\mathrm{sig}}\left(f_{q_{o}}\right)\right|^{2}+\left|R_{\text {ici }}\left(f_{q_{o}}\right)\right|^{2}
\end{aligned}
$$

where $\left|R_{\mathrm{sig}}\right|^{2}$ is the reference subcarrier signal power and $\left|R_{\text {ici }}\right|^{2}$ denotes the total interference power from the other subcarriers $\left(q \neq q_{o}\right)$ to the $q_{o}$ th subcarrier. For a large number of subcarriers $(N \rightarrow \infty)$, the ICI power on the subcarrier is estimated as [55]

$$
\begin{aligned}
\lim _{N \rightarrow \infty} \sum_{\substack{q=0 \\
q \neq q_{o}}}^{2 N-1} \operatorname{sinc}^{2}\left(2 \left(f_{q_{o}}\right.\right. & \left.\left.-f_{q}+f_{d}^{q}\right) \cdot T_{p}\right) \\
& \approx\left(1-\operatorname{sinc}^{2}\left(2 \cdot f_{d}^{q} \cdot T_{p}\right)\right) .
\end{aligned}
$$

In the SAR scenario, multipath signals from a wide target area yield a Doppler spectrum broadening, known as Doppler spread in mobile communications [36]. Therefore, the total ICI power is calculated by multiplying a Doppler distribution function with (19). Now, $f_{d}^{q}$ indicates simple Doppler frequency $f_{d}$. Therefore, the total ICI power $P_{\mathrm{ICI}}$ is given as

$$
P_{\mathrm{ICI}} \triangleq \int_{-\infty}^{\infty}\left(1-\operatorname{sinc}^{2}\left(2 \cdot f_{d} \cdot T_{p}\right)\right) \cdot p_{d}\left(f_{d}\right) \mathrm{d} f_{d} .
$$

\section{REFERENCES}

[1] F. Urlaby and D. Long, Microwave Radar and Radiometric Remote Sensing. Ann Arbor, MI, USA: Univ. of Michigan Press, 2014.

[2] K. Tomiyasu, "Tutorial review of synthetic aperture radar (SAR) with applications to imaging of the ocean surface," Proc. IEEE, vol. 66, no. 5, pp. 563-583, May 1978.

[3] A. Currie and M. A. Brown, "Wide-swath SAR," Proc. Inst. Elect. Eng., vol. 139, no. 2, pp. 122-135, Apr. 1992

[4] W. Wiesbeck, "SDRS: Software defined radar sensors," in Proc. Int. Geosci. Remote Sens. Symp., Sydney, NSW, Australia, Jul. 2001, vol. 7, pp. 3259-3261.

[5] M. Younis, C. Fischer, and W. Wiesbeck, "Digital beamforming in SAR systems," IEEE Trans. Geosci. Remote Sens., vol. 41, no. 7, pp. 17351739, Jul. 2003.

[6] G. Krieger and A. Moreira, "Potentials of digital beamforming in bi- and multistatic SAR," in Proc. Int. Geosci. Remote Sens. Symp., Toulouse, France, Jul. 2003, vol. 1, pp. 527-529.

[7] Z. Li, Z. Bao, H. Wang, and G. Liao, "Performance improvement for constellation SAR using signal processing techniques," IEEE Trans. Aerosp. Electron. Syst., vol. 42, no. 2, pp. 436-452, Apr. 2006.

[8] M. Younis, S. Huber, A. Patyuchenko, F. Bordoni, and G. Krieger, "Performance comparison of reflector- and planar-antenna based digital beamforming SAR," Int. J. Antenna Propag., vol. 2009, pp. 1-13, 2009.

[9] A. Freeman et al., "SweepSAR: Beam-forming on receive using a reflector-phased array feed combination for spaceborne SAR," in Proc. IEEE Radar Conf., Pasadena, CA, USA, May 2009, pp. 1-9.

[10] G. Krieger et al., "Advanced digital beamforming concepts for future SAR systems," in Proc. Int. Geosci. Remote Sens. Symp., Honolulu, HI, USA, Jul. 2010, pp. 245-248.

[11] M. Süß, B. Grafmüller, and R. Zahn, "A novel high-resolution wide-swath SAR system," in Proc. Int. Geosci. Remote Sens. Symp., Sydney, NSW, Australia, Jul. 2001, pp. 1013-1015.
[12] M. Süß and W. Wiesbeck, "Side-looking synthetic aperture radar system," Eur. Patent 1241487 A1, Sep. 18, 2002.

[13] G. Krieger, N. Gebert, and A. Moreira, "Unambiguous SAR signal reconstruction from nonuniform displaced phase center sampling," IEEE Geosci. Remote Sens. Lett., vol. 1, no. 4, pp. 260-264, Oct. 2004.

[14] A. Paulraj and T. Kailath, "Increasing capacity in wireless broadcast systems using distributed transmission/directional reception," U.S. Patent No. 5345 599, Sep. 6, 1994.

[15] E. Fishler et al., "MIMO RADAR: An idea whose time has come," in Proc. IEEE Radar Conf., Philadelphia, PA, USA, Apr. 2004, pp. 71-78.

[16] W. Wang, "MIMO SAR imaging: Potential and challenges," IEEE Aerosp. Electron. Syst. Mag., vol. 28, no. 8, pp. 18-23, Aug. 2013.

[17] D. Cerutti-Maori, I. Sikaneta, J. Klare, and C. Gierull, "MIMO SAR processing for multichannel high-resolution wide-swath radars," IEEE Trans. Geosci. Remote Sens., vol. 52, no. 8, pp. 5034-5055, Aug. 2014.

[18] V. Riche, S. Meric, and E. Pottier, "Study of receiver design in a MIMO SAR configuration," in Proc. EuRAD, Manchester, U.K., Oct. 2011, pp. 297-300

[19] G. Krieger et al., "MIMO-SAR and the orthogonality confusion," in Proc. Int. Geosci. Remote Sens. Symp., Munich, Germany, Jul. 2012, pp. 1533-1536.

[20] M. Younis, G. Krieger, and A. Moreira, "MIMO SAR techniques and trades," in Proc. EuRAD, Nuremberg, Germany, Oct. 2013, pp. 141-144.

[21] P. Huang, H. Li, F. Feng, and M. Huang, "The waveform analysis and signal processing for space-borne MIMO-SAR," in Proc. Eur. Conf. Synthetic Aperture Radar, Nuremberg, Germany, 2012, pp. 579-582.

[22] J. Ender, "Along-track array processing for MIMO-SAR/MTI," in Proc. Eur. Conf. Synthetic Aperture Radar, Friedrichshafen, Germany, Jun. 2008, vol. 4, pp. 67-70.

[23] W. Zhou, H. Kang, J. Wang, and X. Li, "Effect analysis and velocity estimation of moving target based on MIMO SAR system," in Proc. IEEE ICSP, Beijing, China, Oct. 2012, pp. 1752-1755.

[24] D. Pastina, F. Santi, M. Bucciarelli, and P. Lombardo, "2D-MIMO SAR/ISAR imaging of moving targets with reconfigurable formation of platforms," in Proc. Eur. Conf. Synthetic Aperture Radar, Nuremberg, Germany, Apr. 2012, pp. 223-226.

[25] J. Klare, M. Weiss, O. Peters, A. Brenner, and J. Ender, "ARTINO: A new high resolution 3D imaging radar system on an autonomous airborne platform," in Proc. Int. Geosci. Remote Sens. Symp., Denver, CO, USA, Jul. 2006, pp. 3842-3845.

[26] W. Wang, "Space-time coding MIMO-OFDM SAR for high-resolution imaging," IEEE Trans. Geosci. Remote Sens., vol. 49, no. 8, pp. 3094-3104, Aug. 2011.

[27] D. Cristallini, D. Pastina, and P. Lombardo, "Exploiting MIMO SAR potentialities with efficient cross-track constellation configurations for improved range resolution," IEEE Trans. Geosci. Remote Sens., vol. 49, no. 1, pp. 38-53, Jan. 2011.

[28] D. Pastina, M. Bucciarelli, and P. Lombardo, "Multistatic and MIMO distributed ISAR for enhanced cross-range resolution of rotating targets," IEEE Trans. Geosci. Remote Sens., vol. 48, no. 8, pp. 3300-3417, Aug. 2010.

[29] J. Kim, M. Younis, A. Moreira, and W. Wiesbeck, "A novel OFDM chirp waveform scheme for use of multiple transmitters in SAR," IEEE Geosci. Remote Sens. Lett., vol. 10, no. 3, pp. 568-572, May 2013.

[30] G. Krieger, "MIMO SAR: Opportunities and pitfalls," IEEE Trans. Geosci. Remote Sens., vol. 52, no. 5, pp. 2628-2645, May 2014.

[31] A. Oppenheim, A. Willsky, and S. Nawab, Signals and Systems, 2nd ed. Englewood Cliffs, NJ, USA: Prentice-Hall, 1997.

[32] J. C. Curlander and R. N. McDonough, Synthetic Aperture Radar Systems and Signal Processing. Hoboken, NJ, USA: Wiley, 1991.

[33] N. Levanon and E. Mozeson, Radar Signals. Hoboken, NJ, USA: Wiley, 2004.

[34] A. Moreira et al., "A tutorial on synthetic aperture radar," IEEE Geosci. Remote Sens. Mag., vol. 1, no. 1, pp. 6-43, Mar. 2014.

[35] J. Kim, "Multiple-Input Multiple-Output Synthetic Aperture Radar for Multimodal Operation," Ph.D. dissertation, Karlsruhe Inst. Technol. (KIT), Karlsruhe, Germany, 2011.

[36] T. Rappaport, Wireless Communications: Principles and Practice, 2nd ed. Englewood Cliffs, NJ, USA: Prentice-Hall, 2002.

[37] I. G. Cumming and F. H. Wong, Digital Processing of Synthetic Aperture Radar Data. Norwood, MA, USA: Artech House, 2005.

[38] N. Gebert, F. Almeida, and G. Krieger, "Advanced multi-channel SAR imaging-Measured data demonstration," in Proc. IRS, Hamburg, Germany, Sep. 2009, pp. 525-529.

[39] J. Kim, M. Younis, P. Prats-Iraola, M. Gabele, and G. Krieger, "First spaceborne demonstration of digital beamforming for azimuth ambiguity suppression," IEEE Trans. Geosci. Remote Sens., vol. 51, no. 1, pp. 579590, Jan. 2013. 
[40] N. Gebert, "Multi-channel azimuth processing for high-resolution wide-swath SAR imaging," Ph.D. dissertation, Univ. Karlsruhe, Karlsruhe, Germany, 2009.

[41] M. I. Skolnik, Introduction to Radar Systems, 3rd ed. New York, NY, USA: McGraw-Hill, 2001.

[42] C. Schaefer and P. Lopez-Dekker, "Interferometric Ka-band SAR with DBF capability," in Proc. Eur. Conf. Synthetic Aperture Radar, Nuremberg, Germany, Aprl. 2012, pp. 7-10.

[43] J. Ender, C. Gierull, and D. Cerutti-Maori, "Improved space-based moving target indication via alternate transmission and receiver switching," IEEE Trans. Geosci. Remote Sens., vol. 46, no. 12, pp. 3960-1974, Dec. 2008

[44] J. Kim, M. Younis, A. Moreira, and W. Wiesbeck, "A novel OFDM waveform for fully polarimetric SAR data acquisition," in Proc. Eur. Conf. Synthetic Aperture Radar, Aachen, Germany, Jun. 2010, pp. 410-413.

[45] A. Meta, P. Hoogeboom, and L. Lighthart, "Signal processing for FMCW SAR," IEEE Trans. Geosci. Remote Sens., vol. 45, no. 11, pp. 3519-3532, Nov. 2014.

[46] P. Prats-Iraola et al., "On the processing of very high resolution spaceborne SAR data," IEEE Trans. Geosci. Remote Sens, vol. 52, no. 10, pp. 6003-6016, Oct. 2014.

[47] R. Hoctor and S. Kassam, "The unifying role of the coarray in aperture synthesis for coherent and incoherent imaging," Proc. IEEE, vol. 78, no. 4, pp. 735-752, Apr. 1990.

[48] R. J. Sullivan, Microwave Radar Imaging and Advanced Concepts. Norwood, MA, USA: Artech House, 2000.

[49] T. Fritz and M. Eineder, "TerraSAR-X ground segment basic product specification document," German Aerospace Center (DLR), Cologne, Germany, TX-GS-DD-3302, Mar. 2009.

[50] M. Younis, A. Patyuchenko, S. Huber, G. Krieger, and A. Moreira, "A concept for a high performance reflector-based X-band SAR," in Proc. Eur. Conf. Synthetic Aperture Radar, Aachen, Germany, Jun. 2010, pp. 740-743.

[51] A. Moreira et al., "Tandem-L: Monitoring the earth's dynamics with InSAR and Pol-InSAR," presented at the Int. Workshop Appl. Pol-InSAR, Frascati, Italy, Jan., 2009, pp. 1-5.

[52] G. Krieger, H. Fiedler, D. Hounam, and A. Moreira, "Analysis of system concept for bi- and multi-static SAR missions," in Proc. Int. Geosci. Remote Sens. Symp., Toulouse, France, Jul. 2003, vol. 2, pp. 770-772.

[53] D. Garmatyuk and K. Kauffman, "Radar and data communication fusion with UWB-OFDM software-defined system," in Proc. IEEE Int. Conf. Ultra-Wideband, Vancouver, BC, Canada, Sep. 2009, pp. 454-458.

[54] C. Sturm, T. Zwick, and W. Wiesbeck, "An OFDM system concept for joint radar and communications operations," in Proc. IEEE Veh. Technol. Conf., Barcelona, Spain, Apr. 2009, pp. 1-5.

[55] P. Robertson and S. Kaiser, "Analysis of Doppler spread perturbations in OFDM(A) systems," Eur. Trans. Telecommun., vol. 11, no. 6, pp. 585592, Nov./Dec. 2000

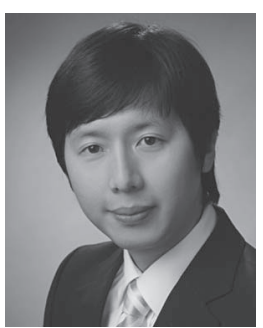

Jung-Hyo Kim (S'04-M'10-13) was born in Seoul, Korea, in 1972. He received the B.Sc. degree in space science and radio engineering from Kyung Hee University, Seoul, in 1999, the M.Sc. degree in electrical engineering from Yonsei University, Seoul, in 2001, and the Dr.-Ing. (Ph.D.) degree in electrical engineering from the Karlsruhe Institute of Technology, Karlsruhe, Germany, in 2011.

From 2001 to 2003, he was an RF System Engineer with the CDMA Laboratory, LG Electronics, Seoul. From 2004 to 2009, he was a Research Scientist with the Institut für Höchstfrequenztechnik und Elektronik, Universität Karlsruhe (TH), Karlsruhe. In 2009, he joined the Microwaves and Radar Institute, German Aerospace Center (DLR), Oberpfaffenhofen, Germany, and participated in advanced synthetic aperture radar (SAR) technique development, including multiple-input multiple-output (MIMO) SAR techniques. Since 2012, he has been a SAR System Engineer with Airbus Defence and Space, Friedrichshafen, Germany. His research interests include radar system engineering, advanced SAR techniques, such as digital beamforming and orthogonal-frequency-division-multiplexing waveform design, and MIMO SAR systems.

Dr. Kim was the recipient of the Second Prize of the Student Paper Competition held during the IEEE International Geoscience and Remote Sensing Symposium 2008, Boston, MA, USA.

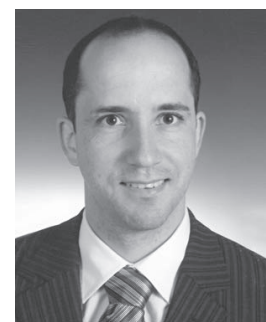

Marwan Younis (S'95-M'05-SM'08) received the B.Sc. degree in electrical engineering from the University of Baghdad, Baghdad, Iraq, in 1992 and the Dipl.-Ing. (M.Sc.) and Dr.-Ing. (Ph.D.) degrees in electrical engineering from Universität Karlsruhe (TH), Karlsruhe, Germany, in 1997 and 2004, respectively.

In 1996, he was an intern at the Jet Propulsion Laboratory (JPL), where he spent a three-month research sabbatical in 2013. From 1998 to 2004 he was a Research Scientist with the Institut für Höchstfrequenztechnik und Elektronik, Universität Karlsruhe (TH). Since 2005, he has been with the Microwaves and Radar Institute, German Aerospace Center (DLR), Oberpfaffenhofen, Germany. He is a Lecturer with Universität Karlsruhe (TH) and Carl-Cranz-Gesellschaft. He is the author and coauthor of about 120 conference papers and 25 reviewed publications. His research fields include synthetic aperture radar (SAR) systems and techniques, multiple-input multiple-output SAR, digital beamforming, SAR performance, calibration, and antennas.

Dr. Younis cochairs the Instrumentation and Future Technologies Geoscience and Remote Sensing Society Technical Committee. He is an Associate Editor for the IEEE GEOSCIENCE AND REMote SENSING LETTER and a Reviewer of IEEE publications. He was the recipient of the Hermann-Billing Award for his Ph.D. thesis in 2005 .

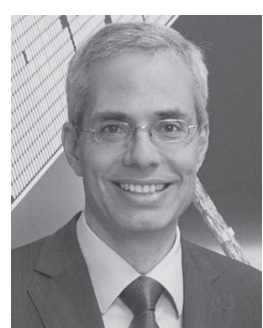

Alberto Moreira (M'92-S'96-F'04) received the B.S.E.E. and M.S.E.E. degrees from the Aeronautical Technological Institute (ITA), Sõo José dos Campos, Brazil, in 1984 and 1986, respectively, and the Eng. Dr. degree (with honors) from the Technical University of Munich, Munich, Germany, in 1993.

From 1996 to 2001, he was the Chief Scientist and Engineer with the SAR Technology Department, German Aerospace Center (DLR), Oberpfaffenhofen, Germany. Since 2001, he has been the Director of the Microwaves and Radar Institute, DLR. The institute contributes to several scientific programs and space projects for actual and future airborne and spaceborne synthetic aperture radar (SAR) missions like TerraSAR-X, TanDEM-X, PAZ, Sentinel-1, SAR-Lupe, BIOMASS, and Tandem-L. The mission TanDEM-X, led by his institute, has successfully started the operational phase in December 2010. He is the Initiator and the Principal Investigator (PI) for this mission. Since 2003, he has also been a Full Professor with the Karlsruhe Institute of Technology, Karlsruhe, Germany, in the field of microwave remote sensing. He has more than 350 publications in international conferences and journals and is the holder of 16 patents in the radar and antenna fields. His professional interests and research areas encompass radar end-to-end system designs and analyses, microwave technologies, techniques and systems, signal and image processing, and remote sensing applications.

Prof. Moreira served as President of the IEEE Geoscience and Remote Sensing (GRS) Society (GRSS) Administrative Committee (2010), was the Founder and the Chair of the GRSS German Chapter (2003-2008), was an Associate Editor for the IEEE GRS LETTERS (2003-2007), and has been an Associate Editor for the IEEE TRANS ACTIONS ON GRS since 2005. He and his colleagues received the GRSS Transactions Prize Paper Awards in 1997, 2001, and 2007 and the IEEE W.R.G. Baker Award in 2012 from the IEEE Board of Directors and were nominated for the Federal Prize for Innovation and Future Technologies of the German President (2012). He was also a recipient of several awards, including the DLR Science Award (1995), IEEE Nathanson Award for the Young Radar Engineer of the Year (1999), the IEEE Kiyo Tomiyasu Field Award (2007), and the IEEE GRSS Distinguished Achievement Award (2014). He served as a member of the Board of Directors of the Information Technology Society of the German Association for Electrical, Electronic and Information Technologies (2003-2008), as Chair of the Scientific and Technical Council of DLR (2009-2011), and as Chair of the DLR Center of Excellence on Synthetic Aperture Radar (2000-2012). He currently serves as Chair of the DLR Center of Excellence on Synthetic Aperture Radar (2014-2016). He has participated as a member of the Technical Program Committee of more than 50 conferences and served as General Chairman for the 2006 European Conference on Synthetic Aperture Radar, as Cochair of the 2007 European Radar Conference, and as General Cochair for the 2012 IEEE International GRS Symposium. Since 2012, he has been serving as Principal Investigator for the Helmholtz Research Alliance "Remote Sensing and Earth System Dynamics," composed of 18 research institutes and 30 associated partners. 


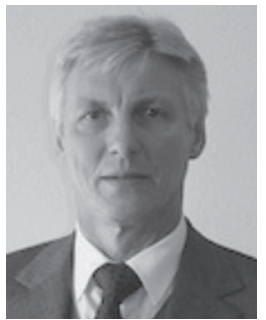

Werner Wiesbeck (SM 87-F 94) received the Dipl.Ing. (M.S.E.E.) and Dr.-Ing. (Ph.D.E.E.) degrees from the Technical University of Munich, Munich, Germany, in 1969 and 1972, respectively.

From 1972 to 1983, he was with AEG-Telefunken in various positions, including the Head of R\&D of the Microwave Division in Flensburg, Germany, and the Marketing Director of the Receiver and Direction Finder Division in Ulm, Germany. During this period, he had product responsibility for millimeterwave radars, receivers, direction finders, and electronic warfare systems. In 1989 and 1994, respectively, he spent a six-month sabbatical at the Jet Propulsion Laboratory, Pasadena, CA, USA. From 1983 to 2007, he was the Director of the Institut für Höchstfrequenztechnik und Elektronik, Universität Karlsruhe (TH), Karlsruhe, Germany, and he is currently a Distinguished Senior Fellow with the Karlsruhe Institute of Technology, Karlsruhe. He is the author and coauthor of several books and of more than 800 publications, a Supervisor of more than 90 Ph.D. students, and a responsible Supervisor of over 600 Diploma/M.S. theses. He is the holder of more than 60 patents. His research topics include antennas, wave propagation, radar, remote sensing, wireless communication, and ultrawideband technologies.

Dr. Wiesbeck was a member of the IEEE Geoscience and Remote Sensing (GRS) Society (GRSS) AdCom (1992-2003), the Chairman of the GRSS Awards Committee (1994, 1998, and 2002), the Executive Vice President of the IEEE GRSS (1998-1999), the President of the IEEE GRSS (2000-2001), an Associate Editor of the IEEE Transactions on Antennas AND PROPAGATION (1996-1999), and a past Treasurer of the IEEE German Section (19871996 and 2003-2007). He was the General Chairman of the 1988 Heinrich Hertz Centennial Symposium and the 1993 Conference on Microwaves and Optics, the Technical Chairman of the 2004 International Millimeter-Wave and Infrared Conference, and the Chairman of the 2006 German Microwave Conference, and he has been a member of the scientific committees and technical program committees of many conferences. For the Carl Cranz Series for Scientific Education, he serves as a permanent Lecturer for radar system engineering, wave propagation, and mobile communication network planning. He is a member of the Advisory Committee of the EU Joint Research Centre (Ispra, Italy), and he is an Advisor to the German Research Council (DFG), to the Federal German Ministry for Research (BMBF), and to industry in Germany. He was the recipient of a number of awards, lately the IEEE Millennium Award, the IEEE GRS Distinguished Achievement Award, the Honorary Doctorate (Dr. h.c.) from the University of Budapest, Budapest, Hungary, the Honorary Doctorate (Dr.-Ing. E.h.) from the University of Duisburg-Essen, Duisburg, Germany, the Honorary Doctorate (Dr.-Ing. E.h.) from Technische Universität Ilmenau, Ilmenau, Germany, and the IEEE Electromagnetics Award in 2008. He is an Honorary Life Member of IEEE GRSS, a member of the Heidelberger Academy of Sciences and Humanities, and a member of the German Academy of Engineering and Technology. He is a member of a number of national and international award committees. 